\title{
Bases for some reciprocity algebras III
}

\author{
Roger Howe and Soo Teck Lee
}

\begin{abstract}
We construct bases for the stable branching algebras for the symmetric pairs $\left(\mathrm{GL}_{2 n}, \mathrm{Sp}_{2 n}\right)$, $\left(\mathrm{Sp}_{2(n+m)}, \mathrm{Sp}_{2 n} \times \mathrm{Sp}_{2 m}\right)$ and $\left(\mathrm{O}_{2 n}, \mathrm{GL}_{n}\right)$. Each basis element is expressed as a sum of products of pfaffians.
\end{abstract}

\section{Introduction}

Let $G$ be a complex classical group and $H$ a symmetric subgroup of $G$, that is, $H$ is the subgroup of fixed points of an involution on $G$. Consider the problem of decomposing an irreducible finite-dimensional representation of $G$ under restriction to $H$. Using classical invariant theory, one can construct an algebra $\mathfrak{A}$ with the following properties: $G$ and a torus $A_{k}$ act on $\mathfrak{A}$ by algebra automorphisms and, under the action of $G \times A_{k}$, we have

$$
\mathfrak{A}=\bigoplus_{i} V_{i} \otimes L_{i}
$$

where each $V_{i}$ is an irreducible $G$ module and each $L_{i}$ is a one-dimensional space on which $A_{k}$ acts by a character $\psi_{i}$. In addition, both the $V_{i}$ and the $\psi_{i}$ are distinct (see, for example, [HTW04]). The subspace $V_{i} \otimes L_{i}$ is the $\psi_{i}$-eigenspace for $A_{k}$ in $\mathfrak{A}$, and since $L_{i}$ is one-dimensional, this subspace can also be regarded as a copy of the irreducible $G$-module $V_{i}$. Thus, if we ignore the action of $A_{k}$, then $\mathfrak{A}$ is a multiplicity free sum of irreducible representations of $G$. We now let $U_{H}$ be the maximal unipotent subgroup of $H$. Then the subalgebra $\mathfrak{A}^{U_{H}}$ of $U_{H}$-invariants in $\mathfrak{A}$ is given by

$$
\mathfrak{A}^{U_{H}}=\bigoplus_{i}\left(V_{i}\right)^{U_{H}} \otimes L_{i} .
$$

This subalgebra carries an action by the maximal torus $A_{H}$ of $H$. If $\phi_{j}$ is a dominant weight for $H$, then the $\phi_{j} \times \psi_{i}$-eigenspace of $A_{H} \times A_{k}$ in $\mathfrak{A}^{U_{H}}$ can be identified with the space of $H$ highest weight vectors of weight $\phi_{j}$ in the $G$-module $V_{i}$. Thus, its dimension is equal to the multiplicity of the irreducible representation of $H$ with highest weight $\phi_{j}$ in $V_{i}$. The representations $V_{i}$ which occur in $\mathfrak{A}$ are of a special type, and they are said to be in the stable range. Thus, the algebra $\mathfrak{A}^{U_{H}}$ encodes all branching information for restricting representations of $G$ in the stable range to $H$. In view of this property, we call $\mathfrak{A}^{U_{H}}$ a stable branching algebra for $(G, H)$. As explained in [HTW04], $\mathfrak{A}^{U_{H}}$ also describes the branching rule for another symmetric pair, so it is also called a reciprocity algebra.

This paper is the third in a series of three which construct explicit bases for stable branching algebras. The most basic case $\left(\mathrm{GL}_{n} \times \mathrm{GL}_{n}, \mathrm{GL}_{n}\right)$ (that is, the case of the $\mathrm{GL}_{n}$ tensor product algebras) was treated in [HTW05b]. The paper [HL06a] deals with two variants of the $\mathrm{GL}_{n}$ tensor product algebras and [HL06b] treats the stable branching algebras for the pairs $\left(\mathrm{GL}_{n}, \mathrm{O}_{n}\right)$, $\left(\mathrm{O}_{n+m}, \mathrm{O}_{n} \times \mathrm{O}_{m}\right)$ and $\left(\mathrm{Sp}_{2 n}, \mathrm{GL}_{n}\right)$. In this paper, we construct bases for the stable branching algebras for the symmetric pairs $\left(\mathrm{GL}_{2 n}, \mathrm{Sp}_{2 n}\right),\left(\mathrm{Sp}_{2(n+m)}, \mathrm{Sp}_{2 n} \times \mathrm{Sp}_{2 m}\right)$ and $\left(\mathrm{O}_{2 n}, \mathrm{GL}_{n}\right)$. Our results

The second named author is partially supported by NUS grant R-146-000-067-112.

This journal is (C) Foundation Compositio Mathematica 2006. 


\section{BASES FOR SOME RECIPROCITY ALGEBRAS III}

will serve two purposes. Firstly, our description of the $H$ highest weight vectors in an irreducible $G$ module $V_{i}$ provides information on how $H$ modules sit inside $V_{i}$. Secondly, these bases provide information about the algebra structure of $\mathfrak{A}^{U_{H}}$. In particular, we will be able to show that the stable branching algebras are flat deformations of the semigroup rings attached to certain lattice cones. In particular, they are flat deformations of toric varieties [HJLTW06].

We follow essentially the same approach as [HL06b]. Each of the stable branching algebras can be realized as an explicit algebra of polynomials on a space of matrices. The bases which we construct are indexed by certain sets of Littlewood-Richardson (LR) tableaux (or ordered pairs of LR tableaux). Each basis element is a specific polynomial, and with respect to a certain monomial order, it has a highest term which allows one to reconstruct the tableau (or the ordered pair of tableaux) from which it came. This shows, in particular, that these bases are SAGBI bases (Subalgebra Analog to Gröbner Bases for Ideals) [RS90, Stu96] for these stable branching algebras (see [HJLTW06]). The main difference from [HL06b] is that each basis element which we construct in this paper is a sum of products of pfaffians (instead of determinants). The main issue is to verify that statements on pfaffians parallel to those on determinants in [HL06b] hold in the new cases. We provide full details on how this can be done in the case of $\left(\mathrm{GL}_{2 n}, \mathrm{Sp}_{2 n}\right)$, but for the other two cases we only state the results.

This paper is arranged as follows. In $\S 2$, we introduce notation for the representations of $\mathrm{GL}_{n}, \mathrm{O}_{n}$ and $\mathrm{Sp}_{2 n}$. We review the definition of pfaffians in $\S 3$. We construct the bases for the stable branching algebras for $\left(\mathrm{GL}_{2 n}, \mathrm{Sp}_{2 n}\right),\left(\mathrm{Sp}_{2(n+m)}, \mathrm{Sp}_{2 n} \times \mathrm{Sp}_{2 m}\right)$ and $\left(\mathrm{O}_{2 n}, \mathrm{GL}_{n}\right)$ in $\S \S 4,5$ and 6 , respectively.

\section{Notation}

\subsection{Representations of $\mathrm{GL}_{n}, \mathrm{O}_{n}$ and $\mathrm{Sp}_{2 n}$}

We use the following standard notation: $\mathrm{GL}_{n}=\mathrm{GL}_{n}(\mathbb{C})$ for the general linear group of invertible $n \times n$ complex matrices; $\mathrm{O}_{n}=\mathrm{O}_{n}(\mathbb{C})$ for the orthogonal group, the subgroup of $\mathrm{GL}_{n}$ which preserves a non-degenerate symmetric bilinear form; and $\mathrm{Sp}_{2 n}=\mathrm{Sp}_{2 n}(\mathbb{C})$, the symplectic group, for the subgroup of $\mathrm{GL}_{2 n}$ which preserves a non-degenerate skew-symmetric bilinear form. We shall also identify a Young diagram $D$ with its sequence of row lengths $D=\left(\lambda_{1}, \ldots, \lambda_{k}\right)$. The number of rows in $D$ is denoted by $r(D)$. Young diagrams will be used to parametrize irreducible representations of the groups $\mathrm{GL}_{n}, \mathrm{O}_{n}$ and $\mathrm{Sp}_{2 n}$ (see, for example, [How95]).

$\mathrm{GL}_{n}$ : Let $A_{\mathrm{GL}_{n}}$ be the diagonal torus in $\mathrm{GL}_{n}$. For Young diagrams $D=\left(\lambda_{1}, \ldots, \lambda_{r}\right)$ and $E=$ $\left(\mu_{1}, \ldots, \mu_{s}\right)$ such that $r+s \leqslant n$, we let $\psi_{n}^{D, E}: A_{\mathrm{GL}_{n}} \rightarrow \mathbb{C}^{\times}$be the character given by

$$
\psi_{n}^{D, E}\left[\operatorname{diag}\left(a_{1}, \ldots, a_{n}\right)\right]=\left[a_{1}^{\lambda_{1}} \cdots a_{r}^{\lambda_{r}}\right]\left[a_{n-s+1}^{-\mu_{s}} \cdots a_{n}^{-\mu_{1}}\right],
$$

and let $\rho_{n}^{D, E}$ be the irreducible representation of $\mathrm{GL}_{n}$ with highest weight $\psi_{n}^{D, E}$. When $E=$ (0), we shall write $\psi_{n}^{D,(0)}$ and $\rho_{n}^{D,(0)}$ simply as $\psi_{n}^{D}$ and $\rho_{n}^{D}$, respectively.

$\mathrm{O}_{n}$ : If $D$ is a Young diagram such that the sum of the lengths of the first two columns of $D$ does not exceed $n$, we let $\sigma_{n}^{D}$ be the irreducible representation of $\mathrm{O}_{n}$ generated by the $\mathrm{GL}_{n}$ highest weight vector in $\rho_{n}^{D}$. If $r(D) \neq n / 2$, the restriction of $\sigma_{n}^{D}$ to $\mathrm{SO}_{n}=\mathrm{SO}_{n}(\mathbb{C})$ is irreducible. The maximal torus $A_{\mathrm{SO}_{n}}$ of $\mathrm{SO}_{n}$ is isomorphic to $\left(\mathbb{C}^{\times}\right)^{m}$, where $m=[n / 2]$. If $D$ is a Young diagram with $r(D)<n / 2$, let $\phi_{n}^{D}: A_{\mathrm{SO}_{n}} \rightarrow \mathbb{C}^{\times}$be the restriction of the character $\psi_{n}^{D}$ to $A_{\mathrm{SO}_{n}}$. Then as a $\mathrm{SO}_{n}$ module, $\sigma_{n}^{D}$ has highest weight $\phi_{n}^{D}$.

$\mathrm{Sp}_{2 n}$ : The diagonal torus $A_{\mathrm{Sp}_{2 n}}$ of $\mathrm{Sp}_{2 n}$ is isomorphic to $\left(\mathbb{C}^{\times}\right)^{n}$. The highest weights and the irreducible finite-dimensional representations of $\mathrm{Sp}_{2 n}$ are parametrized by Young diagrams with at most $n$ rows. If $D$ is such a Young diagram, we denote the corresponding highest weight and representation by $\chi_{2 n}^{D}$ and $\tau_{2 n}^{D}$, respectively. 


\section{R. HOWE AND S. T. LEE}

\subsection{The matrix associated with a LR tableau}

If $E$ is a Young diagram, then by a banal tableau of shape $E$, we mean the tableau obtained by filling each column of $E$ from top to bottom with consecutive positive integers starting from 1.

In [HTW05b], for a given LR tableau (see [Fu97]) $T$ of shape $F / D$ where $D$ and $F$ are Young diagrams and $D$ is contained in $F$, a banal tableau $B T$ and a 'content preserving' map from $T$ to $B T$ are defined, i.e. each cell of $T$ is mapped to a cell in $B T$ with the same value. The map can be visualized as the process of successively removing the 'vertical skew strips' from $T$ and reassembling them into columns of BT. This process is called 'standard peeling'. Thus $T$ is constructed by the reverse process of standard peeling. The contents of $B T$ are moved to the skew diagram one column at a time, starting from the last column of $B T$. If $B T$ has shape $E$, then we say ' $T$ is a LR tableau of shape $F / D$ and content $E$ '. Readers may refer to [HTW05b] for a detailed description of this process. Using standard peeling, we can associate $T$ with the matrix $M(T)=\left(m_{i j}\right)$ of nonnegative integers where $m_{i j}$ is the number of entries from the $j$ th column of $E$ that get put into the $i$ th column of $F / D$.

\section{Review on pfaffians}

Let $A=\left(a_{i j}\right)$ be a $2 n \times 2 n$ skew symmetric complex matrix. The pfaffian of $A$ is defined by [GW98]

$$
\operatorname{Pfaff}(A)=\frac{1}{n ! 2^{n}} \sum_{\sigma \in S_{2 n}} \operatorname{sgn}(\sigma) \prod_{i=1}^{n} a_{\sigma(2 i-1), \sigma(2 i)} .
$$

Here $S_{2 n}$ denote the symmetric group on $\{1,2, \ldots, 2 n\}$. To remove the factor of $1 /\left(n ! 2^{n}\right)$, we let $C(2 n)$ be the subset of $S_{2 n}$ which contains all permutations $\sigma$ such that:

(i) $\sigma(2 i-1)<\sigma(2 i)$ for all $1 \leqslant i \leqslant n$; and

(ii) $\sigma(2 j)<\sigma(2 j+2)$ for all $1 \leqslant j \leqslant n-1$.

Then an elementary argument shows that

$$
\operatorname{Pfaff}(A)=\sum_{\sigma \in C(2 n)} \operatorname{sgn}(\sigma) \prod_{i=1}^{n} a_{\sigma(2 i-1), \sigma(2 i)} .
$$

In this paper, we only consider the pfaffian of those matrices $A$ of the following form:

$$
A=\left(\begin{array}{cc}
B & C \\
-C^{\mathrm{t}} & 0
\end{array}\right)
$$

where $B$ is an $m \times m$ skew-symmetric matrix, $C$ is as $m \times(2 n-m)$ matrix and $n \leqslant m<2 n$. In this case,

$$
\operatorname{Pfaff}(A)=\sum_{\sigma \in C(2 n, m)}(\operatorname{sgn} \sigma) \prod_{i=1}^{m-n} a_{\sigma(2 i-1), \sigma(2 i)} \prod_{j=1}^{2 n-m} a_{\sigma(2 m-2 n+2 j-1), m+j}
$$

where

$$
C(2 n, m)=\{\sigma \in C(2 n): \sigma(2 i-1) \leqslant m \forall 1 \leqslant i \leqslant n\} .
$$

Note that when $m=n$, then

$$
\operatorname{Pfaff}(A)=(-1)^{n(n-1) / 2} \operatorname{det}\left(\begin{array}{cccc}
a_{1, n+1} & a_{1, n+2} & \cdots & a_{1,2 n} \\
a_{2, n+1} & a_{2, n+2} & \cdots & a_{2,2 n} \\
\vdots & \vdots & & \vdots \\
a_{n, n+1} & a_{n, n+2} & \cdots & a_{n, 2 n}
\end{array}\right) .
$$




\section{BASES FOR SOME RECIPROCITY ALGEBRAS III}

\section{A basis for the stable branching algebra for $\left(\mathrm{GL}_{2 n}, \mathrm{Sp}_{2 n}\right)$}

\subsection{The algebra $\mathcal{P}\left(\mathrm{M}_{2 n, k}\right)^{U_{\mathrm{SP}_{2 n}} \times U_{\mathrm{GL}_{k}}}$}

Let $n$ and $k$ be positive integers such that $k \leqslant n$, and let $\mathcal{P}\left(\mathrm{M}_{2 n, k}\right)$ be the algebra of polynomial functions on the space $\mathrm{M}_{2 n, k}=\mathrm{M}_{2 n, k}(\mathbb{C})$ of $2 n \times k$ complex matrices. Let $\mathrm{GL}_{2 n}$ and $\mathrm{GL}_{k}$ act on $\mathcal{P}\left(\mathrm{M}_{2 n, k}\right)$ by the formula:

$$
[(g, h) f](X)=f\left(g^{\mathrm{t} X h}\right), \quad g \in \mathrm{GL}_{2 n}, h \in \mathrm{GL}_{k}, f \in \mathcal{P}\left(\mathrm{M}_{2 n, k}\right), X \in \mathrm{M}_{2 n, k}
$$

and let $\mathrm{Sp}_{2 n}$ act by the restriction of the action of $\mathrm{GL}_{2 n}$. By the $\left(\mathrm{GL}_{2 n}, \mathrm{GL}_{k}\right)$-duality,

$$
\mathcal{P}\left(\mathrm{M}_{2 n, k}\right)=\sum_{r(F) \leqslant k} \rho_{2 n}^{F} \otimes \rho_{k}^{F} .
$$

Let $U_{\mathrm{GL}_{k}}$ and $U_{\mathrm{Sp}_{2 n}}$ denote the standard maximal unipotent subgroups of $\mathrm{GL}_{k}$ and $\mathrm{Sp}_{2 n}$, respectively. Taking $U_{\mathrm{GL}_{k}}$-invariants in $\mathcal{P}\left(\mathrm{M}_{2 n, k}\right)$ gives

$$
\mathcal{P}\left(\mathrm{M}_{2 n, k}\right)^{U_{\mathrm{GL}_{k}}}=\sum_{r(F) \leqslant k} \rho_{2 n}^{F} \otimes\left(\rho_{k}^{F}\right)^{U_{\mathrm{GL}_{k}}} .
$$

This algebra contains one copy of each irreducible representation $\rho_{2 n}^{F}$ of $\mathrm{GL}_{2 n}$ with $r(F) \leqslant k$. We consider the subalgebra of $U_{\mathrm{Sp}_{2 n}}$-invariants in $\mathcal{P}\left(\mathrm{M}_{2 n, k}\right)^{U_{\mathrm{GL}_{k}} \text { : }}$

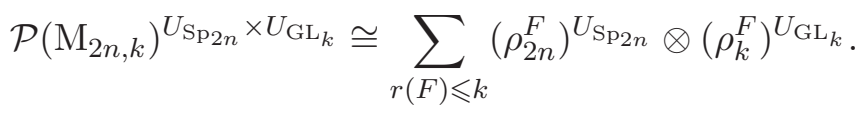

This algebra is a module for $A_{\mathrm{Sp}_{2 n}} \times A_{\mathrm{GL}_{k}}$, where $A_{\mathrm{Sp}_{2 n}}$ and $A_{\mathrm{GL}_{k}}$, are the diagonal torus of $\mathrm{Sp}_{2 n}$ and $\mathrm{GL}_{k}$, respectively. So, we can also write

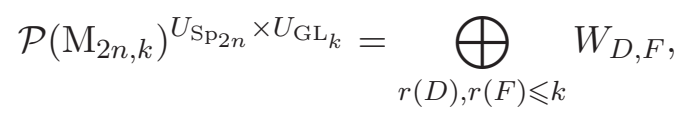

where for each pair $(D, F)$ of Young diagrams with at most $k$ rows, $W_{D, F}$ is the $\chi_{2 n}^{D} \times \psi_{k}^{F}$-eigenspace of $A_{\mathrm{Sp}_{2 n}} \times A_{\mathrm{GL}_{k}}$. The elements in $W_{D, F}$ can be identified with the $\mathrm{Sp}_{2 n}$ highest weight vectors in $\rho_{2 n}^{F}$ with weight $\chi_{2 n}^{D}$. Thus, the algebra structure of $\mathcal{P}\left(\mathrm{M}_{2 n, k}\right)^{U_{\mathrm{Sp}_{2 n}} \times U_{\mathrm{GL}_{k}}}$ carries information on the branching rule from $\mathrm{GL}_{2 n}$ to $\mathrm{Sp}_{2 n}$ for the representations $\rho_{2 n}^{F}$ with $r(F) \leqslant k$. In view of this property, we call $\mathcal{P}\left(\mathrm{M}_{2 n, k}\right)^{U_{\mathrm{Sp}_{2 n}} \times U_{\mathrm{GL}_{k}}}$ a stable branching algebra for $\left(\mathrm{GL}_{2 n}, \mathrm{Sp}_{2 n}\right)$ (see [HTW04]). The goal of this section is to construct a basis for this algebra.

\subsection{The $\mathrm{Sp}_{2 n} \times \mathrm{GL}_{k}$ module structure of $\mathcal{P}\left(\mathrm{M}_{2 n, k}\right)$}

We write a typical element of $\mathrm{M}_{2 n, k}$ as $X=\left(x_{i j}\right)$. For $1 \leqslant i, j \leqslant k$, let

$$
\omega_{i j}(X)=\sum_{a=1}^{n}\left(x_{a i} x_{n+a, j}-x_{n+a, i} x_{a j}\right) .
$$

By the first fundamental theorem of invariant theory for $\mathrm{Sp}_{2 n}$, the algebra $\mathcal{P}\left(\mathrm{M}_{n k}\right)^{\mathrm{Sp}_{2 n}}$ of $\mathrm{Sp}_{2 n}$ invariants in $\mathcal{P}\left(\mathrm{M}_{2 n, k}\right)$ is generated by the $\omega_{i j}$. In fact, since $k \leqslant n$,

$$
\mathcal{P}\left(\mathrm{M}_{2 n, k}\right)^{\mathrm{Sp}_{2 n}}=\mathbb{C}\left[\omega_{i j}: 1 \leqslant i<j \leqslant k\right] \cong \mathcal{P}\left(\bigwedge^{2}\left(\mathbb{C}^{k}\right)\right) \cong \sum_{E \in \mathcal{E}_{k}} \rho_{k}^{E}
$$

as a $\mathrm{GL}_{k}$ module [How95], where

$$
\mathcal{E}_{k}=\left\{\left(\mu_{1}, \mu_{1}, \mu_{2}, \mu_{2}, \ldots, \mu_{m}, \mu_{m}\right): \mu_{1} \geqslant \ldots \geqslant \mu_{m} \geqslant 0,2 m \leqslant k\right\}
$$

is the set of all Young diagrams $E$ such that $E$ has at most $k$ rows and all of its column lengths are even. 
Next, for $1 \leqslant i, j \leqslant k$, we let

$$
\Delta_{i j}=\sum_{a=1}^{n}\left(\frac{\partial^{2}}{\partial x_{a i} \partial x_{n+a, j}}-\frac{\partial^{2}}{\partial x_{n+a, i} \partial x_{a j}}\right),
$$

and

$$
\mathcal{H}\left(\mathrm{M}_{2 n, k}, \mathrm{Sp}_{2 n}\right)=\left\{f \in \mathcal{P}\left(\mathrm{M}_{2 n, k}\right): \Delta_{i j}(f)=0, \forall 1 \leqslant i, j \leqslant k\right\} .
$$

Under the action of $\mathrm{Sp}_{2 n} \times \mathrm{GL}_{k}$ (see [How95]),

$$
\mathcal{H}\left(\mathrm{M}_{2 n, k}, \mathrm{Sp}_{2 n}\right) \cong \sum_{r(D) \leqslant k} \tau_{2 n}^{D} \otimes \rho_{k}^{D}
$$

Since $k \leqslant n$, we have

$$
\mathcal{P}\left(\mathrm{M}_{2 n, k}\right) \cong \mathcal{H}\left(\mathrm{M}_{2 n, k}, \mathrm{Sp}_{2 n}\right) \otimes \mathcal{P}\left(\mathrm{M}_{2 n, k}\right)^{\mathrm{Sp}_{2 n}}
$$

as a $\mathrm{Sp}_{2 n} \times \mathrm{GL}_{k}$ module. It follows from this and the LR rule that

$$
\mathcal{P}\left(\mathrm{M}_{2 n, k}\right) \cong \sum_{r(D), r(F) \leqslant k}\left(\sum_{E \in \mathcal{E}_{k}} c_{E, D}^{F}\right) \tau_{2 n}^{D} \otimes \rho_{k}^{F} .
$$

Here $c_{E, D}^{F}$ is the LR coefficient which counts the number of LR tableaux of shape $F / E$ and content $D$ (see [Fu97]). In particular, this implies that

$$
\operatorname{dim} W_{D, F}=\sum_{E \in \mathcal{E}_{k}} c_{E, D}^{F}
$$

\subsection{The algebra $\mathcal{P}\left(\mathrm{M}_{2 n, k} \oplus \mathrm{AM}_{k}\right)^{U_{\mathrm{Sp}_{2 n}} \times U_{\mathrm{GL}_{k}}}$}

The algebra $\mathcal{P}\left(\mathrm{M}_{2 n, k}\right)^{U_{\mathrm{Sp}_{2 n}} \times U_{\mathrm{GL}_{k}}}$ contains $\mathrm{Sp}_{2 n} \times \mathrm{GL}_{k}$ highest weight vectors. In this subsection, we shall prove that $\mathcal{P}\left(\mathrm{M}_{2 n, k}\right)^{U \mathrm{Sp}_{2 n}} \times U_{\mathrm{GL}_{k}}$ is isomorphic to an algebra of $\mathrm{GL}_{2 n} \times \mathrm{GL}_{k}$ highest weight vectors. This allows us to apply the results of $[\mathrm{HL} 06 \mathrm{~b}, \S 5]$ in our construction of the basis.

Let $\mathrm{AM}_{k}=\mathrm{AM}_{k}(\mathbb{C})$ denote the space of all $k \times k$ skew-symmetric complex matrices. Let $\mathrm{GL}_{2 n} \times$ $\mathrm{GL}_{k}$ act on $\mathrm{M}_{2 n, k} \oplus \mathrm{AM}_{k}$ by

$$
(g, h)(X, \xi)=\left(\left(g^{-1}\right)^{\mathrm{t} X h^{-1}},\left(h^{-1}\right)^{\mathrm{t}} \xi h^{-1}\right), \quad g \in \mathrm{GL}_{2 n}, h \in \mathrm{GL}_{k}, X \in \mathrm{M}_{2 n, k}, \quad \xi \in \mathrm{AM}_{k} .
$$

This extends to an action of $\mathrm{GL}_{2 n} \times \mathrm{GL}_{k}$ on the polynomial algebra $\mathcal{P}\left(\mathrm{M}_{2 n, k} \oplus \mathrm{AM}_{k}\right)$ on $\mathrm{M}_{2 n, k} \oplus \mathrm{AM}_{k}$ in the usual way. Let $\mathcal{P}\left(\mathrm{M}_{2 n, k} \oplus \mathrm{AM}_{k}\right)^{U_{\mathrm{GL}_{2 n}}} \times U_{\mathrm{GL}_{k}}$ be the subalgebra of $U_{\mathrm{GL}_{2 n}} \times U_{\mathrm{GL}_{k}}$-invariants in $\mathcal{P}\left(\mathrm{M}_{2 n, k} \oplus \mathrm{AM}_{k}\right)$. We claim that the algebras $\mathcal{P}\left(\mathrm{M}_{2 n, k}\right)^{U_{\mathrm{Sp}_{2 n}} \times U_{\mathrm{GL}_{k}}}$ and $\mathcal{P}\left(\mathrm{M}_{2 n, k} \oplus \mathrm{AM}_{k}\right)^{U_{\mathrm{GL}_{2 n}} \times U_{\mathrm{GL}_{k}}}$ are isomorphic. In fact, the space $\mathcal{H}\left(\mathrm{M}_{2 n, k}, \mathrm{Sp}_{2 n}\right){ }^{U} \mathrm{Sp}_{2 n}$ of $U_{\mathrm{Sp}_{2 n}}$-invariants in $\mathcal{H}\left(\mathrm{M}_{2 n, k}, \mathrm{Sp}_{2 n}\right)$ coincides with the space $\mathcal{P}\left(\mathrm{M}_{2 n, k}\right)^{U_{\mathrm{GL}_{2 n}}}$ of $U_{\mathrm{GL}_{2 n}}$-invariants in $\mathcal{P}\left(\mathrm{M}_{2 n, k}\right)$. Using this and (4.3), we have

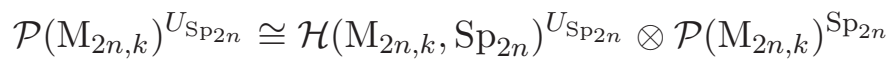

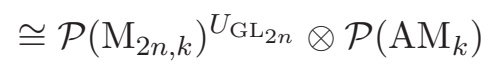

$$
\begin{aligned}
& \cong \mathcal{P}\left(\mathrm{M}_{2 n, k} \oplus \mathrm{AM}_{k}\right)^{U_{\mathrm{GL}_{2 n}}}
\end{aligned}
$$

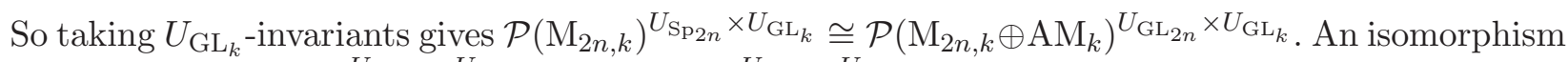
$\alpha: \mathcal{P}\left(\mathrm{M}_{2 n, k} \oplus \mathrm{AM}_{k}\right)^{U_{\mathrm{GL}_{2 n}} \times U_{\mathrm{GL}_{k}}} \rightarrow \mathcal{P}\left(\mathrm{M}_{2 n, k}\right)^{U_{\mathrm{Sp}_{2 n}} \times U_{\mathrm{GL}_{k}}}$ is defined as follows: if $f \in \mathcal{P}\left(\mathrm{M}_{2 n, k} \oplus\right.$ $\left.\mathrm{AM}_{k}\right)^{U_{\mathrm{GL}_{2 n}} \times U_{\mathrm{GL}_{k}}}$ and $X \in \mathrm{M}_{2 n, k}$, then

$$
[\alpha(f)](X)=f(X, \omega(X)) .
$$

Here $\omega(X)=\left(\omega_{i j}(X)\right)$ and $\omega_{i j}(X)$ is defined in (4.1). Observe that $\alpha$ sends the $\psi_{2 n}^{D} \times \psi_{k}^{F}$-eigenspace $\widetilde{W}_{D, F}$ of $A_{\mathrm{GL}_{2 n}} \times A_{\mathrm{GL}_{k}}$ in $\mathcal{P}\left(\mathrm{M}_{2 n, k} \oplus \mathrm{AM}_{k}\right)^{U_{2 n} \times U_{k}}$ to $W_{D, F}$. It follows from this and (4.4) that $\operatorname{dim} \widetilde{W}_{D, F}=\sum_{E \in \mathcal{E}_{k}} c_{E, D}^{F}$. 


\section{4 $\mathrm{GL}_{k}$ highest weight vectors in $\mathcal{P}\left(\mathrm{M}_{2 n, k} \oplus \mathrm{AM}_{k}\right)$}

We denote a typical element in $\mathrm{M}_{2 n, k} \oplus \mathrm{AM}_{k}$ by $(X, \xi)$ where $X=\left(x_{i j}\right)$ and $\xi=\left(\xi_{i j}\right)$. So $\xi_{i j}=-\xi_{j i}$ and $\xi_{i i}=0$ for all $1 \leqslant i, j \leqslant k$. Let $m$ and $p$ be positive integers such that $p \leqslant m \leqslant k$ and $m+p$ is even. Let $[\xi]_{m}$ be the following $m \times m$ submatrix of $\xi$ :

$$
[\xi]_{m}=\left(\begin{array}{cccc}
\xi_{11} & \xi_{12} & \cdots & \xi_{1 m} \\
\xi_{21} & \xi_{22} & \cdots & \xi_{2 m} \\
\vdots & \vdots & & \vdots \\
\xi_{m 1} & \xi_{m 2} & \cdots & \xi_{m m}
\end{array}\right) .
$$

Let $\mathbf{v}_{1}, \ldots, \mathbf{v}_{p} \in \mathbb{C}^{k}$ where, for each $1 \leqslant i \leqslant p$,

$$
\mathbf{v}_{i}=\left(v_{i 1}, v_{i 2}, \ldots, v_{i k}\right) .
$$

We set

and

$$
\left[\mathbf{v}_{1}, \ldots, \mathbf{v}_{p}\right]_{m}=\left(\begin{array}{cccc}
v_{11} & v_{12} & \cdots & v_{1 m} \\
v_{21} & v_{22} & \cdots & v_{2 m} \\
\vdots & \vdots & & \vdots \\
v_{p 1} & v_{p 2} & \cdots & v_{p m}
\end{array}\right)
$$

$$
P_{m}\left(\mathbf{v}_{1}, \ldots, \mathbf{v}_{p}\right)=\operatorname{Pfaff}\left(\begin{array}{c|c}
{[\xi]_{m}} & \left(\left[\mathbf{v}_{1}, \ldots, \mathbf{v}_{p}\right]_{m}\right)^{\mathrm{t}} \\
\hline-\left[\mathbf{v}_{1}, \ldots, \mathbf{v}_{p}\right]_{m} & 0
\end{array} .\right.
$$

Next, for $1 \leqslant i \leqslant 2 n$, let

$$
X_{i}=\left(x_{i 1}, x_{i 2}, \ldots, x_{i k}\right) .
$$

For $1 \leqslant j_{1}, \ldots, j_{p} \leqslant 2 n$, consider the polynomial

$$
P_{m}\left(X_{j_{1}}, \ldots, X_{j_{p}}\right)
$$

on $\mathrm{M}_{2 n, k} \oplus \mathrm{AM}_{k}$.

Lemma 4.4.1. The polynomial $P_{m}\left(X_{j_{1}}, \ldots, X_{j_{p}}\right)$ is a $\mathrm{GL}_{k}$ highest weight vector of weight $\psi_{k}^{\mathbf{1}_{m}}$ in $\mathcal{P}\left(\mathrm{M}_{2 n, k} \oplus \mathrm{AM}_{k}\right)$ where

$$
\mathbf{1}_{m}=(\overbrace{1, \ldots, 1}^{m}, 0, \ldots, 0)
$$

Proof. By (3.1),

$$
P_{m}\left(X_{j_{1}}, \ldots, X_{j_{p}}\right)=\sum_{\sigma \in C(p+m, m)}(\operatorname{sgn} \sigma) \prod_{i=1}^{(m-p) / 2} \xi_{\sigma(2 i-1), \sigma(2 i)} \prod_{b=1}^{p} x_{j_{b}, \sigma(m-p+2 b-1)}
$$

where $C(m+p, m)$ is the subset of the symmetric group $S_{m+p}$ defined in (3.2). From this expression, it is easy to see that $P_{m}\left(X_{j_{1}}, \ldots, X_{j_{p}}\right)$ is a $\mathrm{GL}_{k}$ weight vector of weight $\psi_{k}^{\mathbf{1}_{m}}$.

We consider the derived action of the Lie algebra $\mathfrak{g l}_{k}=\mathfrak{g l}_{k}(\mathbb{C})$ of $\mathrm{GL}_{k}$ on $\mathcal{P}\left(\mathrm{M}_{2 n, k} \oplus \mathrm{AM}_{k}\right)$. Let $E_{a b}$ be the element in $\mathfrak{g l}_{k}$ such that its $(a, b)$ th entry is 1 and 0 elsewhere. Then $E_{a b}$ acts by the differential operator

$$
E_{a b}=\sum_{j=1}^{2 n} x_{j a} \frac{\partial}{\partial x_{j b}}+\sum_{i<b} \xi_{i a} \frac{\partial}{\partial \xi_{i b}}+\sum_{j>b} \xi_{a j} \frac{\partial}{\partial \xi_{b j}} .
$$

We need to show that $E_{a b}\left[P_{m}\left(X_{j_{1}}, \ldots, X_{j_{p}}\right)\right]=0$ for $1 \leqslant a<b \leqslant k$. Note that

$$
E_{a b}\left[P_{m}\left(X_{j_{1}}, \ldots, X_{j_{p}}\right)^{2}\right]=2 P_{m}\left(X_{j_{1}}, \ldots, X_{j_{p}}\right)\left\{E_{a b}\left[P_{m}\left(X_{j_{1}}, \ldots, X_{j_{p}}\right)\right]\right\} .
$$

Thus, it suffices to show that $E_{a b}\left[P_{m}\left(X_{j_{1}}, \ldots, X_{j_{p}}\right)^{2}\right]=0$. 
R. Howe And S. T. LeE

Let

$$
A=\left(\begin{array}{c|c}
{[\xi]_{m}} & \left(\left[X_{j_{1}}, \ldots, X_{j_{p}}\right]_{m}\right)^{\mathrm{t}} \\
\hline-\left[X_{j_{1}}, \ldots, X_{j_{p}}\right]_{m} & 0
\end{array}\right)
$$

Then

$$
\left[P_{m}\left(X_{j_{1}}, \ldots, X_{j_{p}}\right)\right]^{2}=\operatorname{det} A .
$$

First we note that if $b>m$, then from the expression for the operator $E_{a b}$ given in (4.7), it is clear that $E_{a b}(\operatorname{det} A)=0$. So we assume that $1 \leqslant a<b \leqslant m$. For each $1 \leqslant i, j \leqslant m+p$, let $A(i, j)$ be the matrix obtained from $A$ by deleting its $i$ th row and $j$ th column, and for each $1 \leqslant l \leqslant m+p$, let $A[l]$ be the matrix obtained from $A$ by replacing its $l$ th row by its image $R_{l}$ under $E_{a b}$. Specifically,

$$
R_{l}= \begin{cases}\overbrace{0, \ldots, 0, \xi_{l a}}, 0, \ldots, 0) & 1 \leqslant l \leqslant m, l \neq a, b \\ \overbrace{(\overbrace{0, \ldots, 0,0}^{b}, 0, \ldots, 0)}^{b} b, 0, x_{j_{l-m}, a}, 0, \ldots, 0) & m+1 \leqslant l \leqslant m+p,\end{cases}
$$

and

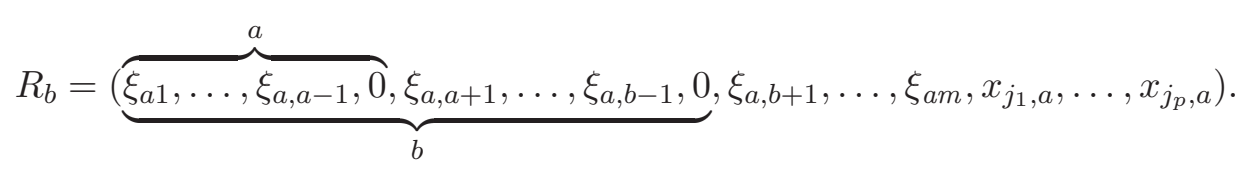

By expanding the determinant of $A[l]$ along its $l$ th row, we obtain

$$
\operatorname{det} A[l]= \begin{cases}(-1)^{b+l} \xi_{l a} \operatorname{det} A(l, b) & 1 \leqslant l \leqslant m, l \neq a, b \\ 0 & l=a \\ -(-1)^{b+l} x_{j_{l-m}, a} \operatorname{det} A(l, b) & m+1 \leqslant l \leqslant m+p .\end{cases}
$$

By adding the $(-1)$ multiple of the $a$ th row of $A[b]$ to its $b$ th row, the $b$ th row becomes

$$
\overbrace{0, \ldots, 0,-\xi_{a b}}^{b}, 0, \ldots, 0)=(\overbrace{0, \ldots, 0, \xi_{b a}}^{b}, 0, \ldots, 0) .
$$

So

$$
\operatorname{det} A[b]=(-1)^{b+l} \xi_{b a} \operatorname{det} A(b, b) .
$$

Consequently,

$$
\begin{aligned}
E_{a b}(\operatorname{det} A) & =\sum_{l=1}^{m+p} \operatorname{det} A[l] \\
& =\sum_{l=1}^{m}(-1)^{b+l} \xi_{l a} \operatorname{det} A(l, b)+\sum_{l=m+1}^{m+p}(-1)^{b+l}\left(-x_{j_{l-m}, a}\right) \operatorname{det} A(l, b) .
\end{aligned}
$$

Observe that the right-hand side of the above equation is the expansion of the determinant of the matrix obtained from $A$ by replacing its $b$ th column by its ath column. Since this matrix has two equal columns,

$$
E_{a b}(\operatorname{det} A)=0
$$




\section{BASES FOR SOME RECIPROCITY ALGEBRAS III}

\subsection{The action by $\mathfrak{g l}_{2 n}$}

We now investigate how the Lie algebra $\mathfrak{g l}_{2 n}$ of $\mathrm{GL}_{2 n}$ acts on the polynomial $P_{m}\left(X_{j_{1}}, X_{j_{2}}, \ldots, X_{j_{p}}\right)$ which is defined in (4.5).

Lemma 4.5.1. If $T \in \mathfrak{g l}_{2 n}$ and $1 \leqslant j_{1}, \ldots, j_{p} \leqslant 2 n$, then

$$
T\left[P_{m}\left(X_{j_{1}}, X_{j_{2}}, \ldots, X_{j_{p}}\right)\right]=\sum_{l=1}^{p} P_{m}\left(X_{j_{1}}, \ldots, X_{j_{l-1}}, T\left(X_{j_{l}}\right), X_{j_{l+1}}, \ldots, X_{j_{p}}\right),
$$

where, for each $1 \leqslant l \leqslant p$,

$$
T\left(X_{j_{l}}\right)=\left(T\left(x_{j_{l}, 1}\right), T\left(x_{j_{l}, 2}\right), \ldots, T\left(x_{j_{l}, k}\right)\right) .
$$

Proof. Using the formula for $P_{m}\left(X_{j_{1}}, X_{j_{2}}, \ldots, X_{j_{p}}\right)$ given in (4.6), we obtain

$$
\begin{aligned}
T & \left.P_{m}\left(X_{j_{1}}, X_{j_{2}}, \ldots, X_{j_{p}}\right)\right] \\
& =\sum_{\sigma \in C(p+m, m)}(\operatorname{sgn} \sigma) \prod_{a=1}^{(m-p) / 2} \xi_{\sigma(2 a-1), \sigma(2 a)} T\left(\prod_{b=1}^{p} x_{j_{b}, \sigma(m-p+2 b-1)}\right) \\
& =\sum_{\sigma \in C(p+m, m)}(\operatorname{sgn} \sigma) \prod_{a=1}^{(m-p) / 2} \xi_{\sigma(2 a-1), \sigma(2 a)}\left[\sum_{l=1}^{p} T\left(x_{j_{l}, \sigma(m-p+2 l-1)}\right) \prod_{\substack{1 \leqslant b \leqslant p \\
b \neq l}} x_{j_{b}, \sigma(m-p+2 b-1)}\right] \\
& =\sum_{l=1}^{p}\left\{\sum_{\sigma \in C(p+m, m)}(\operatorname{sgn} \sigma) \prod_{a=1}^{(m-p) / 2} \xi_{\sigma(2 a-1), \sigma(2 a)}\left[T\left(x_{j_{l}, \sigma(m-p+2 l-1)}\right)\right] \prod_{\substack{1 \leqslant b \leqslant p \\
b \neq l}} x_{j_{b}, \sigma(m-p+2 b-1)}\right\} \\
& =\sum_{l=1}^{p} P_{m}\left(X_{j_{1}}, \ldots, X_{j_{l-1}}, T\left(X_{j_{l}}\right), X_{j_{l+1}}, \ldots, X_{j_{p}}\right) .
\end{aligned}
$$

Corollary 4.5.2. Let $1 \leqslant p \leqslant m \leqslant k$ and let $\left\{\varepsilon_{1}, \ldots, \varepsilon_{2 n}\right\}$ be the standard basis for $\mathbb{C}^{2 n}$. Then the linear map

$$
\Phi: \bigwedge^{p} \mathbb{C}^{2 n} \rightarrow \mathcal{P}\left(\mathrm{M}_{2 n, k} \oplus \mathrm{AM}_{k}\right)
$$

specified by

$$
\Phi\left(\varepsilon_{j_{1}} \wedge \cdots \wedge \varepsilon_{j_{p}}\right)=P_{m}\left(X_{j_{1}}, \ldots, X_{j_{p}}\right)
$$

is a $\mathrm{GL}_{2 n}$ isomorphism from $\bigwedge^{p} \mathbb{C}^{2 n}$ onto its image.

Proof. First we note that the linear map $\left(\mathbb{C}^{2 n}\right)^{p} \rightarrow \mathcal{P}\left(\mathrm{M}_{2 n, k} \oplus \mathrm{AM}_{k}\right)$ specified by

$$
\left(\varepsilon_{j_{1}}, \ldots, \varepsilon_{j_{p}}\right) \rightarrow P_{m}\left(X_{j_{1}}, \ldots, X_{j_{p}}\right)
$$

is alternating. In fact, if $s<t$ and we switch the positions of $X_{j_{s}}$ and $X_{j_{t}}$ in $P_{m}\left(X_{j_{1}}, \ldots, X_{j_{p}}\right)$, then we obtain

$$
\begin{aligned}
P_{m}\left(X_{j_{1}}, \ldots, X_{j_{t}}, \ldots, X_{j_{s}}, \ldots, X_{j_{p}}\right)= & \sum_{\sigma \in C(p+m, m)}(\operatorname{sgn} \sigma)\left[\prod_{i=1}^{(m-p) / 2} \xi_{\sigma(2 i-1), \sigma(2 i)}\right] \\
& \times x_{j_{t}, \sigma(m-p+2 s-1)} x_{j_{s}, \sigma(m-p+2 t-1)} \prod_{\substack{1 \leqslant b \leqslant p \\
b \neq s, t}} x_{j_{b}, \sigma(m-p+2 b-1)} .
\end{aligned}
$$


R. Howe And S. T. LeE

Now for each $\sigma \in C(p+m, m)$, we let $\tilde{\sigma}=\sigma \circ \tau$ where $\tau$ is the transposition $(m-p+2 s-1$, $m-p+2 t-1)$. Then

$$
\begin{aligned}
P_{m} & \left(X_{j_{1}}, \ldots, X_{j_{t}}, \ldots, X_{j_{s}}, \ldots, X_{j_{p}}\right) \\
= & \sum_{\tilde{\sigma} \in C(p+m, m)}(\operatorname{sgn} \tilde{\sigma})(\operatorname{sgn} \tau)\left[\prod_{i=1}^{(m-p) / 2} \xi_{\tilde{\sigma}(2 i-1), \tilde{\sigma}(2 i)]}\right. \\
& \times x_{j_{t}, \tilde{\sigma}(m-p+2 t-1)} x_{j_{s}, \tilde{\sigma}(m-p+2 s-1)} \prod_{\substack{1 \leqslant b \leqslant p \\
b \neq s, t}} x_{j_{b}, \tilde{\sigma}(m-p+2 b-1)} \\
= & -P_{m}\left(X_{j_{1}}, \ldots, X_{j_{s}}, \ldots, X_{j_{t}}, \ldots, X_{j_{p}}\right) .
\end{aligned}
$$

Hence, the linear map $\Phi$ exists. By Lemma 4.5.1, $\Phi$ is a $\mathrm{GL}_{2 n}$ map. Since $\bigwedge^{p} \mathbb{C}^{2 n}$ is an irreducible $\mathrm{GL}_{2 n}$ module, $\Phi$ is a $\mathrm{GL}_{2 n}$ isomorphism from $\bigwedge^{p} \mathbb{C}^{2 n}$ onto its image.

Corollary 4.5.3. The function $P_{m}\left(X_{1}, \ldots, X_{p}\right)$ is a joint $\mathrm{GL}_{2 n} \times \mathrm{GL}_{k}$ highest weight vector of weight $\psi_{2 n}^{\mathbf{1}_{p}} \times \psi_{k}^{\mathbf{1}_{m}}$.

Proof. This is because $\varepsilon_{1} \wedge \cdots \wedge \varepsilon_{p}$ is the $\mathrm{GL}_{2 n}$ highest weight vector in $\wedge^{p} \mathbb{C}^{2 n}$ of weight $\psi_{2 n}^{\mathbf{1}_{p}}$ and $P_{m}\left(X_{1}, \ldots, X_{p}\right)=\Phi\left(\varepsilon_{1} \wedge \cdots \wedge \varepsilon_{p}\right)$.

\section{6 $\mathrm{GL}_{2 n} \times \mathrm{GL}_{k}$ highest weight vectors in $\mathcal{P}\left(\mathrm{M}_{2 n, k} \oplus \mathrm{AM}_{k}\right)$}

Recall that for fixed $D$ and $F$, the $\psi_{2 n}^{D} \times \psi_{k}^{F}$-eigenspace $\widetilde{W}_{D, F}$ in $\mathcal{P}\left(\mathrm{M}_{2 n, k} \oplus \mathrm{AM}_{k}\right)^{U_{2 n} \times U_{k}}$ has dimension $\sum_{E \in \mathcal{E}_{k}} c_{E, D}^{F}$. We now let

$$
\Omega(D, F)
$$

be the set of all ordered pairs $(E, T)$ where $E \in \mathcal{E}_{k}$ and $T$ is a LR tableau of shape $F / E$ and content $D$. Then the number of elements in $\Omega(D, F)$ coincides with $\operatorname{dim} \widetilde{W}_{D, F}$. We shall use the set $\Omega(D, F)$ to index a basis for $\widetilde{W}_{D, F}$.

We now fix $(E, T) \in \Omega(D, F)$ and will construct a $\mathrm{Sp}_{2 n} \times \mathrm{GL}_{k}$ highest weight vector $\zeta_{(E, T)}$ in $\mathcal{P}\left(\mathrm{M}_{2 n, k} \oplus \mathrm{AM}_{k}\right)$ with weight $\psi_{2 n}^{D} \times \psi_{k}^{F}$. Let

$$
\begin{aligned}
D^{\mathrm{t}} & =\left(d_{1}, \ldots, d_{r}\right) \\
E^{\mathrm{t}} & =\left(e_{1}, \ldots, e_{s}\right) \\
F^{\mathrm{t}} & =\left(f_{1}, \ldots, f_{t}\right),
\end{aligned}
$$

the conjugate diagrams of $D, E$ and $F$, respectively. For each $1 \leqslant i \leqslant t$, let

$$
p_{i}=f_{i}-e_{i} .
$$

Recall that $T$ is constructed by the reverse process of standard peeling (see $\S 2.2)$. Let $M(T)=\left(m_{i j}\right)$ be the $t \times r$ matrix where $m_{i j}$ is the number of elements from the $j$ th column of $D$ get put into the $i$ th column of $F$. For $1 \leqslant i \leqslant t$ and $1 \leqslant j \leqslant r$, let

$$
a_{i j}=m_{i j}+m_{i+1, j}+\cdots+m_{t j}
$$

and

$$
a_{t+1, j}=0 .
$$

Then the entries from the $j$ th column of $D$ get put into the $i$ th column of $F$ are

$$
a_{i+1, j}+1, a_{i+1, j}+2, \ldots, a_{i, j} .
$$

We now let $\boldsymbol{\sigma}=\left(\sigma_{1}, \ldots, \sigma_{r}\right) \in S_{d_{1}} \times \cdots \times S_{d_{r}}$. Here $S_{d_{i}}$ denotes the symmetric group on $\left\{1, \ldots, d_{i}\right\}$. 


\section{BASES FOR SOME RECIPROCITY ALGEBRAS III}

For each $1 \leqslant i \leqslant t$, let $\mathcal{X}_{i}(\boldsymbol{\sigma})$ be the $p_{i} \times f_{i}$ matrix

$$
\mathcal{X}_{i}(\boldsymbol{\sigma})=\left(\begin{array}{c}
\mathcal{X}_{i 1}\left(\sigma_{1}\right) \\
\mathcal{X}_{i 2}\left(\sigma_{2}\right) \\
\vdots \\
\mathcal{X}_{i r}\left(\sigma_{r}\right)
\end{array}\right)
$$

where for each $j, \mathcal{X}_{i j}\left(\sigma_{l}\right)$ is the $m_{i l} \times f_{i}$ matrix defined by

$$
\mathcal{X}_{i j}\left(\sigma_{j}\right)=\left(\begin{array}{cccc}
x_{\sigma_{j}\left(a_{i+1, j}+1\right), 1} & x_{\sigma_{j}\left(a_{i+1, j}+1\right), 2} & \cdots & x_{\sigma_{j}\left(a_{i+1, j}+1\right), f_{i}} \\
x_{\sigma_{j}\left(a_{i+1, j}+2\right), 1} & x_{\sigma_{j}\left(a_{i+1, j}+2\right), 2} & \cdots & x_{\sigma_{j}\left(a_{i+1, j}+2\right), f_{i}} \\
\vdots & \vdots & & \vdots \\
x_{\sigma_{j}\left(a_{i, j}\right), 1} & x_{\sigma_{j}\left(a_{i, j}\right), 2} & \cdots & x_{\sigma_{j}\left(a_{i, j}\right), f_{i}}
\end{array}\right) .
$$

Set

$$
\zeta_{(E, T)}=\sum_{\boldsymbol{\sigma} \in S_{d_{1}} \times \cdots \times S_{d_{r}}}\left(\prod_{i=1}^{r} \operatorname{sgn}\left(\sigma_{i}\right)\right) \cdot\left[\prod_{i=1}^{t} \operatorname{Pfaff}\left(\begin{array}{c|c}
{[\xi]_{f_{i}}} & {\left[\mathcal{X}_{i}(\boldsymbol{\sigma})\right]^{\mathrm{t}}} \\
\hline-\mathcal{X}_{i}(\boldsymbol{\sigma}) & 0
\end{array}\right)\right] .
$$

Lemma 4.6.1. The polynomial $\zeta_{(E, T)}$ defined in (4.10) is a $\mathrm{GL}_{2 n} \times \mathrm{GL}_{k}$ highest weight vector in $\mathcal{P}\left(\mathrm{M}_{2 n, k} \oplus \mathrm{AM}_{k}\right)$ of weight $\psi_{2 n}^{D} \times \psi_{k}^{F}$.

Proof. It is easy to verify that the weight of $\zeta_{(E, T)}$ is $\psi_{2 n}^{D} \times \psi_{k}^{F}$. That $\zeta_{(E, T)}$ is a $\mathrm{GL}_{k}$ highest weight vector of weight $\psi_{k}^{F}$ follows from Lemma 4.4.1. To see that $\zeta_{(E, T)}$ is a $\mathrm{GL}_{2 n}$ highest weight vector, let

$$
\Phi: \bigwedge^{p_{1}}\left(\mathbb{C}^{2 n}\right) \otimes \bigwedge^{p_{2}}\left(\mathbb{C}^{2 n}\right) \otimes \cdots \otimes \bigwedge^{p_{t}}\left(\mathbb{C}^{2 n}\right) \longrightarrow \mathcal{P}\left(\mathrm{M}_{2 n, k} \oplus \mathrm{AM}_{k}\right)
$$

be the $\mathrm{GL}_{2 n}$ map defined by

$$
\Phi\left(u_{1} \otimes \cdots \otimes u_{t}\right)=\Phi_{1}\left(u_{1}\right) \cdots \Phi_{t}\left(u_{t}\right)
$$

where for each $1 \leqslant i \leqslant t, u_{i} \in \bigwedge^{p_{i}}\left(\mathbb{C}^{2 n}\right)$ and $\Phi_{i}: \bigwedge^{p_{i}}\left(\mathbb{C}^{2 n}\right) \rightarrow \mathcal{P}\left(\mathrm{M}_{2 n, k} \oplus \mathrm{AM}_{k}\right)$ is the $\mathrm{GL}_{2 n}$-map defined in Corollary 4.5.2. We now observe that $\zeta_{(E, T)}$ is the image of the highest weight vector $\Gamma_{N}$ given in [HL06b, Lemma 6.2.1], where the matrix $N$ is the transpose of $M(T)$. So it is a $\mathrm{GL}_{2 n}$ highest weight vector of weight $\psi_{2 n}^{D}$.

\subsection{The leading monomial of $\zeta_{(E, T)}$}

Recall that typical elements of $\mathrm{M}_{2 n, k}$ and $\mathrm{AM}_{k}$ are written as $X=\left(x_{i j}\right)$ and $\xi=\left(\xi_{i j}\right)$, respectively. We define a monomial ordering $\tau_{1}$ on $\mathcal{P}\left(\mathrm{M}_{2 n, k} \oplus \mathrm{AM}_{k}\right)$ as follows: it is the graded lexicographic order [CLO97] such that

$$
\begin{aligned}
\xi_{12} & >\xi_{13}>\cdots>\xi_{1 k}>\xi_{23}>\cdots>\xi_{2 n}>\xi_{34}>\cdots>\xi_{k-1, k} \\
& >x_{11}>x_{12}>\cdots>x_{1 k}>x_{21}>\cdots>x_{2 n, k} .
\end{aligned}
$$

We shall compute the leading monomial of $\zeta_{(E, T)}$ with respect to $\tau_{1}$.

We first consider the function

$$
P_{m}\left(X_{j_{1}}, \ldots, X_{j_{p}}\right)=\operatorname{Pfaff}\left(\begin{array}{c|c}
{[\xi]_{m}} & \left(\left[X_{j_{1}}, \ldots, X_{j_{p}}\right]_{m}\right)^{\mathrm{t}} \\
\hline-\left[X_{j_{1}}, \ldots, X_{j_{p}}\right]_{m} & 0
\end{array}\right)
$$

which is a building block of the highest weight vector $\zeta_{(E, T)}$. We note that in the expansion of $P_{m}\left(X_{j_{1}}, \ldots, X_{j_{p}}\right)$ given in (4.6), exactly $(m-p) / 2$ entries in each term need to be chosen from the submatrix $[\xi]_{m}$. It is clear from the definition of $\tau_{1}$ that $\xi_{12} \xi_{23} \cdots \xi_{m-p-1, m-p}$ has the highest possible order among all possible choices. 
R. Howe And S. T. LeE

Lemma 4.7.1. Let $\tilde{P}_{m}\left(X_{j_{1}}, \ldots, X_{j_{p}}\right)$ be the sum of all of the terms in $P_{m}\left(X_{j_{1}}, \ldots, X_{j_{p}}\right)$ containing the factor $\xi_{12} \xi_{23} \cdots \xi_{m-p-1, m-p}$. Then

$$
\tilde{P}_{m}\left(X_{j_{1}}, \ldots, X_{j_{p}}\right)=(-1)^{p(p-1) / 2}\left(\xi_{12} \xi_{23} \cdots \xi_{m-p-1, m-p}\right)\left|\begin{array}{cccc}
x_{j_{1}, m-p+1} & x_{j_{1}, m-p+2} & \cdots & x_{j_{1}, m} \\
x_{j_{2}, m-p+1} & x_{j_{2}, m-p+2} & \cdots & x_{j_{2}, m} \\
\vdots & \vdots & & \vdots \\
x_{j_{p}, m-p+1} & x_{j_{p}, m-p+2} & \cdots & x_{j_{p}, m}
\end{array}\right| .
$$

Proof. By (4.6),

$$
P_{m}\left(X_{j_{1}}, \ldots, X_{j_{p}}\right)=\sum_{\sigma \in C(p+m, m)}(\operatorname{sgn} \sigma) \prod_{i=1}^{(m-p) / 2} \xi_{\sigma(2 i-1), \sigma(2 i)} \prod_{b=1}^{p} x_{j_{b}, \sigma(m-p+2 b-1)}
$$

From this, it is clear that if $\sigma \in C(2 n, m)$, then

$$
\xi_{12} \xi_{23} \cdots \xi_{m-p-1, m-p}=\prod_{i=1}^{(m-p) / 2} \xi_{\sigma(2 i-1), \sigma(2 i)}
$$

if and only if

$$
\sigma(j)=j, \quad j=1, \ldots, m-p .
$$

For such a permutation $\sigma$, there exists a unique permutation $\tau \in S_{p}$ such that

$$
\sigma(m-p+2 j-1)=m-p+\tau(j), \quad j=1, \ldots, p .
$$

Conversely, any permutation $\tau \in S_{p}$ uniquely determines an element $\sigma$ of $C(m+p, m)$ with the property $\sigma(j)=j$ for $j=1, \ldots, m-p$. In addition,

$$
\operatorname{sgn} \sigma=(-1)^{p(p-1) / 2} \operatorname{sgn} \tau \text {. }
$$

It follows that

$$
\begin{aligned}
\tilde{P}_{m}\left(X_{j_{1}}, \ldots, X_{j_{p}}\right) & =(-1)^{p(p-1) / 2}\left(\xi_{12} \xi_{23} \cdots \xi_{m-p-1, m-p}\right) \\
& \sum_{\tau \in S_{p}}(\operatorname{sgn} \tau) \prod_{b=1}^{p} x_{j_{b}, m-p+\tau(b)} \\
& =(-1)^{p(p-1) / 2}\left(\xi_{12} \xi_{23} \cdots \xi_{m-p-1, m-p}\right)\left|\begin{array}{cccc}
x_{j_{1}, m-p+1} & x_{j_{1}, m-p+2} & \cdots & x_{j_{1}, m} \\
x_{j_{2}, m-p+1} & x_{j_{2}, m-p+2} & \cdots & x_{j_{2}, m} \\
\vdots & \vdots & & \vdots \\
x_{j_{p}, m-p+1} & x_{j_{p}, m-p+2} & \cdots & x_{j_{p}, m}
\end{array}\right| .
\end{aligned}
$$

Recall that $E$ is the Young diagram given in (4.9). Let

$$
\xi_{E}=\prod_{j=1}^{s}\left(\xi_{12} \xi_{23} \cdots \xi_{e_{j}-1, e_{j}}\right)
$$

and consider the sum of all of the terms in $\zeta_{(E, T)}$ which contain $\xi_{E}$ as a factor. It is of the form

$$
\xi_{E} \cdot\left(\zeta_{(E, T)}\left[\xi_{E}\right]\right)
$$

where $\zeta_{(E, T)}\left[\xi_{E}\right]$ is a polynomial in the variables $\left(x_{i j}\right)$. Note that the leading monomial of $\zeta_{(E, T)}$ is the product of $\xi_{E}$ and the leading monomial of $\zeta_{(E, T)}\left[\xi_{E}\right]$.

Lemma 4.7.2. The leading monomial of $\zeta_{(E, T)}\left[\mu_{E}\right]$ is given by

$$
x_{T}=\prod_{\mathbf{b} \in T} x_{c(\mathbf{b}) a(\mathbf{b})}
$$


where for each box $\mathbf{b}$ in $T, a(\mathbf{b})$ is the row of $F$ in which the box $\mathbf{b}$ lies and $c(\mathbf{b})$ is the entry in $\mathbf{b}$. Consequently, the leading monomial of $\zeta_{(E, T)}$ is $x_{T} \xi_{E}$.

Proof. We first set up some notation. For each $1 \leqslant i \leqslant t$, let $\hat{\mathcal{X}}_{i}(\boldsymbol{\sigma})$ be the $p_{i} \times p_{i}$ matrix

$$
\hat{\mathcal{X}}_{i}(\boldsymbol{\sigma})=\left(\begin{array}{c}
\hat{\mathcal{X}}_{i 1}\left(\sigma_{1}\right) \\
\hat{\mathcal{X}}_{i 2}\left(\sigma_{2}\right) \\
\vdots \\
\hat{\mathcal{X}}_{i r}\left(\sigma_{r}\right)
\end{array}\right)
$$

where, for each $j, \hat{\mathcal{X}}_{i j}\left(\sigma_{j}\right)$ is the $m_{i j} \times p_{i}$ matrix

$$
\hat{\mathcal{X}}_{i j}\left(\sigma_{j}\right)=\left(\begin{array}{cccc}
x_{\sigma_{j}\left(a_{i+1, j}+1\right), e_{i}+1} & x_{\sigma_{j}\left(a_{i+1, j}+1\right), e_{i}+2} & \cdots & x_{\sigma_{j}\left(a_{i+1, j}+1\right), f_{i}} \\
x_{\sigma_{j}\left(a_{i+1, j}+2\right), e_{i}+1} & x_{\sigma_{j}\left(a_{i+1, j}+2\right), e_{i}+2} & \cdots & x_{\sigma_{j}\left(a_{i+1, j}+2\right), f_{i}} \\
\vdots & \vdots & & \vdots \\
x_{\sigma_{j}\left(a_{i, j}\right), e_{i+1}} & x_{\sigma_{j}\left(a_{i, j}\right), e_{i+2}} & \cdots & x_{\sigma_{j}\left(a_{i, j}\right), f_{i}}
\end{array}\right) .
$$

Note that $\hat{\mathcal{X}}_{i}(\boldsymbol{\sigma})$ is obtained from the matrix $\mathcal{X}_{i}(\boldsymbol{\sigma})$ by removing its first $e_{i}$ columns. It is now clear from the expression of $\zeta_{(E, T)}$ given in (4.10) and Lemma 4.7.1 that

$$
\zeta_{(E, T)}\left[\xi_{E}\right]= \pm \sum_{\boldsymbol{\sigma} \in S_{d_{1}} \times \cdots \times S_{d_{r}}}\left(\prod_{i=1}^{r} \operatorname{sgn}\left(\sigma_{i}\right)\right) \prod_{j=1}^{t} \operatorname{det} \hat{\mathcal{X}}_{j}(\boldsymbol{\sigma})
$$

We now need some results of [HTW05b] and [HL06b]. In the paper [HTW05b], a polynomial $\delta_{T}(Y)$ in the variables $Y=\left(y_{i j}\right)$ is defined in (3.4). An ordering on the set of monomials in the variables $y_{i j}$ is defined in $\S 3.4 .1$, and the leading monomial of $\delta_{T}(Y)$ with respect to this monomial ordering is given in Lemma 3.2. On the other hand, an expansion formula for $\delta_{T}(Y)$ is given in [HL06b, Lemma 5.7.2]. We observe that if we identify $x_{i j}$ with the variable $y_{j i}$, then a comparison of (4.15) and the formula given in [HL06b, Lemma 5.7.2] reveals that $\zeta_{(E, T)}\left[\xi_{E}\right]$ coincides with a multiple of $\delta_{T}(Y)$. In addition, under this identification, the monomial orderings defined in [HTW05b] and $\tau_{1}$ are identical. Consequently, the leading monomial of $\zeta_{(E, T)}\left[\xi_{E}\right]$ is the image of the leading monomial of $\delta_{T}(Y)$. Thus, the lemma follows.

\subsection{A basis for $\mathcal{P}\left(\mathrm{M}_{2 n, k}\right)^{U_{\mathrm{Sp}_{2 n}} \times U_{\mathrm{GL}_{k}}}$}

We are now ready to state and prove the main theorem of this section.

ThEOREM 4.8.1. The set

$$
\bigcup_{r(D), r(F) \leqslant k}\left\{\zeta_{(E, T)}:(E, T) \in \Omega(D, F)\right\}
$$

is a basis for $\mathcal{P}\left(\mathrm{M}_{2 n, k} \oplus \mathrm{AM}_{k}\right)^{U_{\mathrm{GL}_{2 n}} \times U_{\mathrm{GL}_{k}}}$. Thus, its image in $\mathcal{P}\left(\mathrm{M}_{2 n, k}\right)^{U_{\mathrm{Sp}_{2 n}} \times U_{\mathrm{GL}_{k}}}$ is a basis for $\mathcal{P}\left(\mathrm{M}_{2 n, k}\right)^{U_{\mathrm{Sp}_{2 n}} \times U_{\mathrm{GL}_{k}}}$.

Proof. By Lemma 4.7.2, the leading monomial of $\zeta_{(E, T)}$ is $x_{T} \xi_{E}$. Now $\xi_{E}$ determines the diagram $E$, $x_{T}$ determines the diagram $D$, and $\xi_{E}$ and $x_{T}$ together determine the diagram $F$ and the tableau $T$. This shows that $(D, F, E, T) \rightarrow x_{T} \xi_{E}$ is one-to-one. Since the highest weight vectors $\zeta_{(E, T)}$ have distinct leading monomials, they form a linearly independent set. Moreover, for fixed $D$ and $F$, the number of the highest weight vectors we obtain in this way is equal to the dimension of the $\psi_{2 n}^{D} \times \psi_{k}^{F}$-eigenspace $\widetilde{W}_{D, F}$ of $A_{\mathrm{GL}_{2 n}} \times A_{\mathrm{GL}_{k}}$ in $\mathcal{P}\left(\mathrm{M}_{2 n, k} \oplus \mathrm{AM}_{k}\right)^{U_{\mathrm{GL}_{2 n}} \times U_{\mathrm{GL}_{k}}}$. Hence, they form a basis for this eigenspace. It follows that by varying the diagrams $D$ and $F$ we obtain a basis for $\mathcal{P}\left(\mathrm{M}_{2 n, k} \oplus \mathrm{AM}_{k}\right)^{U_{\mathrm{GL}_{2 n}} \times U_{\mathrm{GL}_{k}}}$. 


\section{R. Howe AND S. T. LeE}

\section{A basis for the stable branching algebras for $\left(\operatorname{Sp}_{2(n+m)}, \mathbf{S p}_{2 n} \times \mathbf{S p}_{2 m}\right)$}

In this section, we construct a basis for the stable branching algebra for the symmetric pair $\left(\mathrm{Sp}_{2(n+m)}, \mathrm{Sp}_{2 n} \times \mathrm{Sp}_{2 m}\right)$. Since the construction is similar to the case of $\left(\mathrm{O}_{n+m}, \mathrm{O}_{n} \times \mathrm{O}_{m}\right)$ which has been done in [HL06b], we omit most of the details.

\subsection{The subgroup $\mathrm{Sp}_{2 n} \times \mathrm{Sp}_{2 m}$ of $\mathrm{Sp}_{2(n+m)}$}

Recall that $\mathrm{Sp}_{2(n+m)}$ is the subgroup of $\mathrm{GL}_{2 n+2 m}$ which leaves the symplectic form $\langle\cdot, \cdot\rangle$ on $\mathbb{C}^{2 n+2 m}$ invariant, where for $\mathbf{a}=\left(a_{1}, \ldots, a_{2 n+2 m}\right), \mathbf{b}=\left(b_{1}, \ldots, b_{2 n+2 m}\right) \in \mathbb{C}^{2 n+2 m}$,

$$
\langle\mathbf{a}, \mathbf{b}\rangle=\sum_{i=1}^{n+m}\left(a_{i} b_{n+m+i}-a_{n+m+i} b_{i}\right) .
$$

Let $\left\{\varepsilon_{1}, \ldots, \varepsilon_{2 n+2 m}\right\}$ be the standard basis for $\mathbb{C}^{2 n+2 m}$. Let $W_{1}$ and $W_{2}$ be the subspaces of $\mathbb{C}^{2 n+2 m}$ spanned by $\left\{\varepsilon_{1}, \ldots, \varepsilon_{n}, \varepsilon_{n+m+1}, \ldots, \varepsilon_{2 n+m}\right\}$ and by $\left\{\varepsilon_{n+1}, \ldots, \varepsilon_{n+m}, \varepsilon_{2 n+m+1}, \ldots, \varepsilon_{2 n+2 m}\right\}$, respectively. Then the subgroup of all elements in $\operatorname{Sp}_{2(n+m)}$ which leave both $W_{1}$ and $W_{2}$ stable is isomorphic to $\mathrm{Sp}_{2 n} \times \mathrm{Sp}_{2 m}$. We abuse notation and denote this subgroup also by $\mathrm{Sp}_{2 n} \times \mathrm{Sp}_{2 m}$.

\subsection{The algebra $\mathcal{R}(\mathcal{V})^{U_{\mathrm{Sp}_{2 n}} \times U_{\mathrm{Sp}_{2 m}} \times U_{\mathrm{GL}_{k}}}$}

Let $k \leqslant \min (n, m)$ and let $\mathrm{M}_{2(n+m), k}=\mathrm{M}_{2(n+m), k}(\mathbb{C})$ be the space of all $2(n+m) \times k$ complex matrices. For $1 \leqslant i, j \leqslant k$ and $T \in \mathrm{M}_{2(n+m), k}$, we let

$$
\xi_{i j}(T)=\left\langle T_{i}, T_{j}\right\rangle,
$$

where $T_{i}$ and $T_{j}$ are the $i$ th and the $j$ th column of $T$, respectively. Let

$$
\mathcal{V}=\left\{T \in \mathrm{M}_{2(n+m), k}: \xi_{i j}(T)=0 \forall 1 \leqslant i, j \leqslant k\right\},
$$

and let $\mathcal{R}(\mathcal{V})$ be the algebra of regular functions on $\mathcal{V}$. Let $\mathrm{Sp}_{2(n+m)} \times \mathrm{GL}_{k}$ act on $\mathcal{R}(\mathcal{V})$ by

$$
[(g, h) . f](T)=f\left(g^{\mathrm{t}} T h\right), \quad g \in \mathrm{Sp}_{2(n+m)}, h \in \mathrm{GL}_{k}, f \in \mathcal{R}(\mathcal{V}), T \in \mathcal{V} .
$$

Then as a $\mathrm{Sp}_{2(n+m)} \times \mathrm{GL}_{k}$ module [How95],

$$
\mathcal{R}(\mathcal{V}) \cong \sum_{r(H) \leqslant k} \tau_{2(n+m)}^{H} \otimes \rho_{k}^{H} .
$$

Let $U_{\mathrm{GL}_{k}}, U_{\mathrm{Sp}_{2 n}}$ and $U_{\mathrm{Sp}_{2 m}}$ be the maximal unipotent subgroups of $\mathrm{GL}_{k}, \mathrm{Sp}_{2 n}$ and $\mathrm{Sp}_{2 m}$, respectively. Taking $U_{\mathrm{GL}_{k}}$ invariants in $\mathcal{R}(\mathcal{V})$ gives

$$
\mathcal{R}(\mathcal{V})^{U_{\mathrm{GL}}} \cong \sum_{r(H) \leqslant k} \tau_{2(n+m)}^{H} \otimes\left(\rho_{k}^{H}\right)^{U_{\mathrm{GL}_{k}}} .
$$

This algebra contains one copy of each irreducible representation $\tau_{2 n+2 m}^{H}$ of $\operatorname{Sp}_{2(n+m)}$ with $r(H) \leqslant k$.

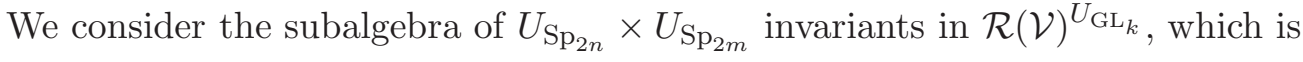

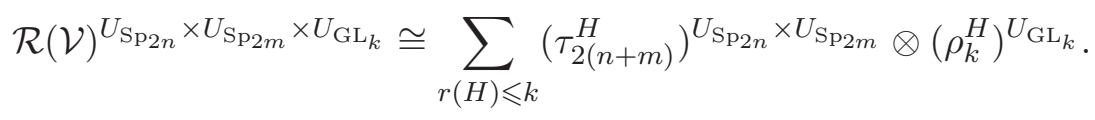

This algebra encodes all information on the branching rule from $\operatorname{Sp}_{2(n+m)}$ to $\mathrm{Sp}_{2 n} \times \mathrm{Sp}_{2 m}$ for all representations $\tau_{2(n+m)}^{H}$ with $r(H) \leqslant k$. In view of this property, we call $\mathcal{R}(\mathcal{V})^{U_{\mathrm{Sp}_{2 n}} \times U_{\mathrm{Sp}_{2 m}} \times U_{\mathrm{GL}_{k}}}$ a stable branching algebra for $\left(\mathrm{Sp}_{2(n+m)}, \mathrm{Sp}_{2 n} \times \mathrm{Sp}_{2 m}\right)$ (see [HTW04]). The goal of this section is to construct a basis for this algebra. 


\subsection{The algebra $\mathcal{P}\left(\mathrm{M}_{2 n, k} \oplus \mathrm{M}_{2 m, k} \oplus \mathrm{AM}_{k}\right)^{U_{\mathrm{GL}_{2 n}} \times U_{\mathrm{GL}_{2 m}} \times U_{\mathrm{GL}_{k}}}$}

As in the case of $\left(\mathrm{O}_{n+m}, \mathrm{O}_{n} \times \mathrm{O}_{m}\right)$, we can replace the stable branching algebra $\mathcal{R}(\mathcal{V})^{U \mathrm{~S}_{\mathrm{p}_{2}} \times U_{\mathrm{S}_{2} m} \times U_{\mathrm{GL}_{k}}}$ by a subalgebra of a polynomial algebra which we now describe. Let $\mathrm{GL}_{2 n} \times \mathrm{GL}_{2 m} \times \mathrm{GL}_{k}$ act on $\mathrm{M}_{2 n, k} \oplus \mathrm{M}_{2 m, k} \oplus \mathrm{AM}_{k}$ by

$$
\left(g_{1}, g_{2}, h\right)(X, Y, N)=\left(\left(g_{1}^{-1}\right)^{\mathrm{t}} X h^{-1},\left(g_{2}^{-1}\right)^{\mathrm{t}} Y h^{-1},\left(h^{-1}\right)^{\mathrm{t}} N h^{-1}\right)
$$

where $\left(g_{1}, g_{2}, h\right) \in \mathrm{GL}_{2 n} \times \mathrm{GL}_{2 m} \times \mathrm{GL}_{k}, X \in \mathrm{M}_{2 n, k}, Y \in \mathrm{M}_{2 m, k}$ and $N \in \mathrm{AM}_{k}$. This induces an action of $\mathrm{GL}_{2 n} \times \mathrm{GL}_{2 m} \times \mathrm{GL}_{k}$ on the polynomial algebra $\mathcal{P}\left(\mathrm{M}_{2 n, k} \oplus \mathrm{M}_{2 m, k} \oplus \mathrm{AM}_{k}\right)$. We consider the subalgebra

$$
\mathcal{P}\left(\mathrm{M}_{2 n, k} \oplus \mathrm{M}_{2 m, k} \oplus \mathrm{AM}_{k}\right)^{U_{\mathrm{GL}_{2 n}} \times U_{\mathrm{GL}_{2 m}} \times U_{\mathrm{GL}_{k}}}
$$

of $U_{\mathrm{GL}_{2 n}} \times U_{\mathrm{GL}_{2 m}} \times U_{\mathrm{GL}_{k}}$-invariants in $\mathcal{P}\left(\mathrm{M}_{2 n, k} \oplus \mathrm{M}_{2 m, k} \oplus \mathrm{AM}_{k}\right)$. This algebra is isomorphic to $\mathcal{R}(\mathcal{V})^{U \mathrm{Sp}_{2 n}} \times U_{\mathrm{Sp}_{2 m}} \times U_{\mathrm{GL}_{k}}$. We now describe an isomorphism

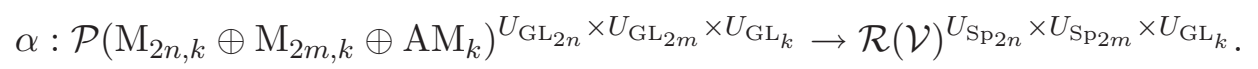

Recall that, in $\S 5.1$, we defined the symplectic form $\langle\cdot, \cdot\rangle$ and the subspaces $W_{1}$ and $W_{2}$ of $\mathbb{C}^{2 n+2 m}$. Note that $\mathbb{C}^{2 n+2 m}=W_{1} \oplus W_{2}$, and for $i=1,2$, we let $\pi_{i}: \mathbb{C}^{2 n+2 m} \rightarrow W_{i}$ be the corresponding linear projection. Let $\langle\cdot, \cdot\rangle_{1}$ and $\langle\cdot, \cdot\rangle_{2}$ be the restrictions of $\langle\cdot, \cdot\rangle$ to $W_{1}$ and $W_{2}$, respectively. For $1 \leqslant i, j \leqslant k$ and $T \in \mathrm{M}_{2(n+m), k}$, let

$$
\nu_{i j}(T)=\left\langle\pi_{1}\left(T_{i}\right), \pi_{1}\left(T_{j}\right)\right\rangle_{1}-\left\langle\pi_{2}\left(T_{i}\right), \pi_{2}\left(T_{j}\right)\right\rangle_{2}
$$

where $T_{i}$ and $T_{j}$ are the $i$ th and the $j$ th column of $T$. Now if

$$
f \in \mathcal{P}\left(\mathrm{M}_{2 n, k} \oplus \mathrm{M}_{2 m, k} \oplus \mathrm{AM}_{k}\right)^{U_{\mathrm{GL}_{2 n}} \times U_{\mathrm{GL}_{2 m}} \times U_{\mathrm{GL}_{k}}}
$$

and $T \in \mathcal{V}$, then $\alpha f$ is the function on $\mathcal{V}$ defined by

$$
(\alpha f)(T)=f(X, Y, N)
$$

where

$$
T=\left(\begin{array}{c}
X_{1} \\
Y_{1} \\
X_{2} \\
Y_{2}
\end{array}\right), \quad X=\left(\begin{array}{c}
X_{1} \\
X_{2}
\end{array}\right) \in \mathrm{M}_{2 n, k}, \quad Y=\left(\begin{array}{c}
Y_{1} \\
Y_{2}
\end{array}\right) \in \mathrm{M}_{2 m, k}, \quad \text { and } \quad N=\left(\nu_{i j}(T)\right) .
$$

\subsection{An index set for the basis}

We shall construct a basis for the algebra

$$
\mathcal{P}\left(\mathrm{M}_{2 n, k} \oplus \mathrm{M}_{2 m, k} \oplus \mathrm{AM}_{k}\right)^{U_{\mathrm{GL}_{2 n}} \times U_{\mathrm{GL}_{2 m}} \times U_{\mathrm{GL}_{k}} .}
$$

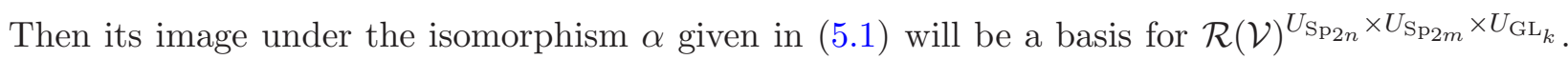
First we note that under the action by $A_{\mathrm{GL}_{2 n}} \times A_{\mathrm{GL}_{2 m}} \times A_{\mathrm{GL}_{k}}$,

$$
\mathcal{P}\left(\mathrm{M}_{2 n, k} \oplus \mathrm{M}_{2 m, k} \oplus \mathrm{AM}_{k}\right)^{U_{\mathrm{GL}_{2 n}} \times U_{\mathrm{GL}_{2 m}} \times U_{\mathrm{GL}_{k}}}=\bigoplus_{r(D), r(E), r(H) \leqslant k} \widetilde{W}_{D, E, H},
$$

where $\widetilde{W}_{D, E, H}$ is the $\psi_{2 n}^{D} \times \psi_{2 m}^{E} \times \psi_{k}^{H}$-eigenspace of $A_{\mathrm{GL}_{2 n}} \times A_{\mathrm{GL}_{2 m}} \times A_{\mathrm{GL}_{k}}$. It is clear that the image of $\widetilde{W}_{D, E, H}$ under $\alpha$ is precisely the $\chi_{2 n}^{D} \times \chi_{2 m}^{E} \times \psi_{k}^{H}$-eigenspace $W_{D, E, H}$ of $A_{\mathrm{Sp}_{2 n}} \times A_{\mathrm{Sp}_{2 m}} \times A_{\mathrm{GL}_{k}}$

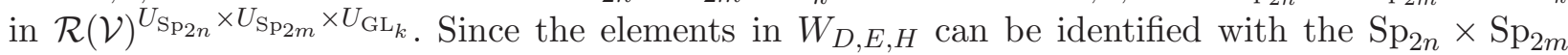
highest weight vectors in $\tau_{2 n+2 m}^{H}$ with weight $\chi_{2 n}^{D} \times \chi_{2 m}^{E}$, the dimension of $W_{D, E, H}$ is equal to the multiplicity of the $\tau_{2 n}^{D} \otimes \tau_{2 m}^{E}$ in $\tau_{2 n+2 m}^{H}$. This is given by (see [HTW05a])

$$
\operatorname{dim} \widetilde{W}_{D, E, H}=\operatorname{dim} W_{D, E, H}=\sum_{F \in \mathcal{E}_{k}, r(G) \leqslant k} c_{F, D}^{G} c_{G, E}^{H} .
$$


We now let

$$
\Omega(D, E, H)
$$

be the set of all ordered 4-tuples $\left(F, G, T_{1}, T_{2}\right)$ such that $F \in \mathcal{E}_{k}, G$ is a Young diagram with at most $k$ rows, and $T_{1}$ (respectively $T_{2}$ ) is a LR tableaux of shape $G / F$ (respectively $H / G$ ) with content $D$ (respectively $E$ ). Note that the number of elements in $\Omega(D, E, H)$ coincides with $\operatorname{dim} \widetilde{W}_{D, E, H}$, so we will use it to index a basis for $\widetilde{W}_{D, E, H}$.

\subsection{Highest weight vectors in $\widetilde{W}_{D, E}, H$}

We now fix $\left(F, G, T_{1}, T_{2}\right) \in \Omega(D, E, H)$ and construct a $\mathrm{GL}_{2 n} \times \mathrm{GL}_{2 m} \times \mathrm{GL}_{k}$ highest weight vector $\omega_{\left(F, G, T_{1}, T_{2}\right)}$ of weight $\psi_{2 n}^{D} \times \psi_{2 m}^{E} \times \psi_{k}^{H}$.

First, suppose that the conjugate diagrams of $D, E, F, G$ and $H$ are given by

$$
\begin{aligned}
D^{\mathrm{t}} & =\left(d_{1}, \ldots, d_{r}\right) \\
E^{\mathrm{t}} & =\left(e_{1}, \ldots, e_{s}\right) \\
F^{\mathrm{t}} & =\left(f_{1}, \ldots, f_{t}\right) \\
G^{\mathrm{t}} & =\left(g_{1}, \ldots, g_{u}\right) \\
H^{\mathrm{t}} & =\left(h_{1}, \ldots, h_{v}\right) .
\end{aligned}
$$

Consider the reverse process of standard peeling in the construction of $T_{1}$ and $T_{2}$ (see $\S 2.2$ ). Let $M\left(T_{1}\right)=\left(m_{i j}^{(1)}\right)$ and $M\left(T_{2}\right)=\left(m_{i j}^{(2)}\right)$ where:

- $m_{i j}^{(1)}$ is the number of elements from the $j$ th column of $D$ get put into the $i$ th column of $G$;

- $m_{i j}^{(2)}$ is the number of elements from the $j$ th column of $E$ get put into the $i$ th column of $H$.

For $1 \leqslant i \leqslant u$ and $1 \leqslant j \leqslant r$, let

$$
a_{i j}^{(1)}=m_{i j}^{(1)}+m_{i+1, j}^{(1)}+\cdots+m_{u j}^{(1)},
$$

and

and, for $1 \leqslant i \leqslant v$ and $1 \leqslant j \leqslant s$, let

$$
a_{u+1, j}^{(1)}=0
$$

$$
a_{i j}^{(2)}=m_{i j}^{(2)}+m_{i+1, j}^{(2)}+\cdots+m_{v j}^{(2)},
$$

and

$$
a_{v+1, j}^{(2)}=0
$$

Then:

- the entries from the $j$ th column of $D$ that get put into the $i$ th column of $G / F$ are

$$
a_{i+1, j}^{(1)}+1, a_{i+1, j}^{(1)}+2, \ldots, a_{i, j}^{(1)}
$$

- the entries from the $j$ th column of $E$ that get put into the $i$ th column of $H / G$ are

$$
a_{i+1, j}^{(2)}+1, a_{i+1, j}^{(2)}+2, \ldots, a_{i, j}^{(2)} .
$$

We are now ready to define the highest weight vector $\omega_{\left(F, G, T_{1}, T_{2}\right)}$. Write a typical element $\mathrm{M}_{2 n, k} \oplus$ $\mathrm{M}_{2 m, k} \oplus \mathrm{AM}_{k}$ as $(X, Y, N)$ where $X=\left(x_{i j}\right), Y=\left(y_{i j}\right)$ and $N=\left(\nu_{i j}\right)$. Let $\boldsymbol{\sigma}=\left(\sigma_{1}, \ldots, \sigma_{r}\right) \in$ $S_{d_{1}} \times \cdots \times S_{d_{r}}$ and $\tau=\left(\tau_{1}, \ldots, \tau_{s}\right) \in S_{e_{1}} \times \cdots \times S_{e_{s}}$. For each $1 \leqslant i \leqslant u$, let

$$
p_{i}=g_{i}-f_{i}
$$


and $\mathcal{X}_{i}(\boldsymbol{\sigma})$ be the $p_{i} \times h_{i}$ matrix

$$
\mathcal{X}_{i}(\boldsymbol{\sigma})=\left(\begin{array}{c}
\mathcal{X}_{i 1}\left(\sigma_{1}\right) \\
\mathcal{X}_{i 2}\left(\sigma_{2}\right) \\
\vdots \\
\mathcal{X}_{i r}\left(\sigma_{r}\right)
\end{array}\right)
$$

where, for each $1 \leqslant j \leqslant r$,

$$
\mathcal{X}_{i j}\left(\sigma_{j}\right)=\left(\begin{array}{cccc}
x_{\sigma_{j}\left(a_{i+1, j}^{(1)}+1\right), 1} & x_{\sigma_{j}\left(a_{i+1, j}^{(1)}+1\right), 2} & \cdots & x_{\sigma_{j}\left(a_{i+1, j}^{(1)}+1\right), h_{i}} \\
x_{\sigma_{j}\left(a_{i+1, j}^{(1)}+2\right), 1} & x_{\sigma_{j}\left(a_{i+1, j}^{(1)}+2\right), 2} & \cdots & x_{\sigma_{j}\left(a_{i+1, j}^{(1)}+2\right), h_{i}} \\
\vdots & \vdots & & \vdots \\
x_{\sigma_{j}\left(a_{i, j}^{(1)}\right), 1} & x_{\sigma_{j}\left(a_{i, j}^{(1)}\right), 2} & \cdots & x_{\sigma_{j}\left(a_{i, j}^{(1)}\right), h_{i}}
\end{array}\right),
$$

and, for $1 \leqslant i \leqslant v$, let

$$
q_{i}=h_{i}-g_{i}
$$

and $\mathcal{Y}_{i}(\boldsymbol{\tau})$ be the $q_{i} \times h_{i}$ matrix

$$
\mathcal{Y}_{i}(\boldsymbol{\tau})=\left(\begin{array}{c}
\mathcal{Y}_{i 1}\left(\tau_{1}\right) \\
\mathcal{Y}_{i 2}\left(\tau_{2}\right) \\
\vdots \\
\mathcal{Y}_{i s}\left(\tau_{s}\right)
\end{array}\right)
$$

where, for each $1 \leqslant l \leqslant s$,

$$
\mathcal{Y}_{i l}\left(\tau_{l}\right)=\left(\begin{array}{cccc}
y_{\tau_{l}\left(a_{i+1, l}^{(2)}+1\right), 1} & y_{\tau_{l}\left(a_{i+1, l}^{(2)}+1\right), 2} & \cdots & y_{\tau_{l}\left(a_{i+1, l}^{(2)}+1\right), h_{i}} \\
y_{\tau_{l}\left(a_{i+1, l}^{(2)}+2\right), 1} & y_{\tau_{l}\left(a_{i+1, l}^{(2)}+2\right), 2} & \cdots & y_{\tau_{l}\left(a_{i+1, l}^{(2)}+2\right), h_{i}} \\
\vdots & \vdots & & \vdots \\
y_{\tau_{l}\left(a_{i, l}^{(2)}\right), 1} & y_{\tau_{l}\left(a_{i, l}^{(2)}\right), 2} & \cdots & y_{\tau_{l}\left(a_{i, l}^{(2)}\right), h_{i}}
\end{array}\right) .
$$

We also let, for $1 \leqslant m \leqslant k$,

Finally, we let

$$
[\nu]_{m}=\left(\begin{array}{cccc}
\nu_{11} & \nu_{12} & \cdots & \nu_{1 m} \\
\nu_{21} & \nu_{22} & \cdots & \nu_{2 m} \\
\vdots & \vdots & & \vdots \\
\nu_{m 1} & \nu_{m 2} & \cdots & \nu_{m, m}
\end{array}\right)
$$

$$
\begin{aligned}
\omega_{\left(F, G, T_{1}, T_{2}\right)}= & \sum_{\substack{\boldsymbol{\sigma} \in S_{d_{1}} \times \cdots \times S_{d_{r}} \\
\boldsymbol{\tau} \in S_{e_{1}} \times \cdots \times S_{e_{s}}}}\left(\prod_{i=1}^{r} \operatorname{sgn}\left(\sigma_{i}\right)\right)\left(\prod_{j=1}^{s} \operatorname{sgn}\left(\tau_{j}\right)\right) \\
& \times\left[\prod_{i=1}^{v} \operatorname{Pfaff}\left(\begin{array}{c|c|c}
{[\nu]_{h_{i}}} & {\left[\mathcal{X}_{i}(\boldsymbol{\sigma})\right]^{\mathrm{t}}} & {\left[\mathcal{Y}_{i}(\boldsymbol{\tau})\right]^{\mathrm{t}}} \\
\hline-\mathcal{X}_{i}(\boldsymbol{\sigma}) & 0 & 0 \\
\hline-\mathcal{Y}_{i}(\boldsymbol{\sigma}) & 0 & 0
\end{array}\right)\right] .
\end{aligned}
$$

This is a $\mathrm{GL}_{2 n} \times \mathrm{GL}_{2 m} \times \mathrm{GL}_{k}$ highest weight vector in $\mathcal{P}\left(\mathrm{M}_{2 n, k} \oplus \mathrm{M}_{2 m, k} \oplus \mathrm{AM}_{k}\right)$ of weight $\psi_{2 n}^{D} \times$ $\psi_{2 m}^{E} \times \psi_{k}^{H}$.

\subsection{The leading monomial of $\omega_{\left(F, G, T_{1}, T_{2}\right)}$}

Recall that typical elements of $\mathrm{M}_{2 n, k}, \mathrm{M}_{2 m, k}$ and $\mathrm{AM}_{k}$ are written $X=\left(x_{i j}\right), Y=\left(y_{i j}\right)$ and $N=\left(\nu_{i j}\right)$, respectively. We define a monomial ordering $\tau_{2}$ on $\mathcal{P}\left(\mathrm{M}_{2 n, k} \oplus \mathrm{M}_{2 m, k} \oplus \mathrm{AM}_{k}\right)$ as follows: 
R. Howe And S. T. LeE

it is the graded lexicographic order [CLO97] such that

$$
\begin{aligned}
\nu_{12} & >\nu_{13}>\cdots>\nu_{1 k}>\nu_{23}>\nu_{24}>\cdots>\nu_{k-1, k} \\
& >x_{11}>x_{12}>\cdots>x_{1 k}>x_{21}>\cdots>x_{2 n, k} \\
& >y_{11}>y_{12}>\cdots>y_{1 k}>y_{21}>\cdots>y_{2 m, k} .
\end{aligned}
$$

Lemma 5.6.1. The leading monomial of $\omega_{\left(F, G, T_{1}, T_{2}\right)}$ with respect to $\tau_{2}$ is given by

$$
\left(\prod_{\mathbf{b}_{1} \in T_{1}} x_{c_{1}\left(\mathbf{b}_{1}\right) a_{1}\left(\mathbf{b}_{1}\right)}\right)\left(\prod_{\mathbf{b}_{2} \in T_{2}} y_{c_{2}\left(\mathbf{b}_{2}\right) a_{2}\left(\mathbf{b}_{2}\right)}\right)\left(\prod_{j=1}^{t} \prod_{i=1}^{f_{j} / 2} \nu_{2 i-1,2 i}\right)
$$

where:

(i) for each box $\mathbf{b}_{1}$ in $T_{1}, a_{1}\left(\mathbf{b}_{1}\right)$ is the row of $G$ in which the box $\mathbf{b}_{1}$ lies and $c_{1}\left(\mathbf{b}_{1}\right)$ is the entry in $\mathbf{b}_{1}$; and

(ii) for each box $\mathbf{b}_{2}$ in $T_{2}, a_{2}\left(\mathbf{b}_{2}\right)$ is the row of $H$ in which the box $\mathbf{b}_{2}$ lies and $c_{2}\left(\mathbf{b}_{2}\right)$ is the entry in $\mathbf{b}_{2}$.

The proof, which we omit, is similar to the proof of Lemma 8.8.1 in [HL06b]. An immediate consequence of this lemma is that the leading monomials of the $\omega_{\left(F, G, T_{1}, T_{2}\right)}$ are all distinct. In particular, it implies the following.

TheOREM 5.6.2. The set

$$
\bigcup_{r(D), r(E), r(H) \leqslant k}\left\{\omega_{\left(F, G, T_{1}, T_{2}\right)}:\left(F, G, T_{1}, T_{2}\right) \in \Omega(D, E, H)\right\}
$$

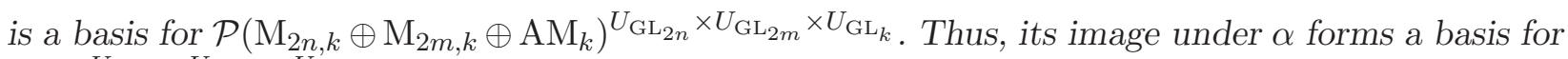
$\mathcal{R}(\mathcal{V})^{U_{\mathrm{Sp}_{2 n}} \times U_{\mathrm{Sp}_{2 m}} \times U_{\mathrm{GL}_{k}}}$.

\section{A basis for the stable branching algebras for $\left(\mathrm{O}_{2 n}, \mathrm{GL}_{n}\right)$}

In this section, we construct a basis for the stable branching algebra for the symmetric pair $\left(\mathrm{O}_{2 n}, \mathrm{GL}_{n}\right)$. Since the construction is very similar to the case of $\left(\mathrm{Sp}_{2 n}, \mathrm{GL}_{n}\right)$ which has been done in [HL06b], we omit most of the details.

\subsection{The even orthogonal group}

Let $(\cdot, \cdot)$ be the symmetric bilinear form on $\mathbb{C}^{2 n}$ given by

$$
(u, v)=\sum_{i=1}^{2 n} u_{i} v_{2 n-i+1}, \quad u=\left(u_{1}, \ldots, u_{2 n}\right), v=\left(v_{1}, \ldots, v_{2 n}\right) \in \mathbb{C}^{2 n} .
$$

Let

$$
\mathrm{O}_{2 n}=\mathrm{O}_{2 n}(\mathbb{C})=\left\{g \in \mathrm{GL}_{2 n}:(g u, g v)=(u, v), \forall u, v \in \mathbb{C}^{2 n}\right\}
$$

Then

$$
\left\{\left(\begin{array}{cc}
a & 0 \\
0 & J\left(a^{-1}\right)^{\mathrm{t}} J
\end{array}\right): a \in \mathrm{GL}_{n}\right\}
$$

is a subgroup of $\mathrm{O}_{2 n}$ and it is isomorphic to $\mathrm{GL}_{n}$. Here $J$ is the $n \times n$ matrix with 1 on its antidiagonal and 0 elsewhere. In this section, we abuse notation and denote this subgroup also by $\mathrm{GL}_{n}$. 


\subsection{The algebra $\mathcal{R}(\mathcal{N})^{U_{\mathrm{GL}_{n}} \times U_{\mathrm{GL}_{k}}}$}

Let $2 k \leqslant n$. For $1 \leqslant i, j \leqslant k$ and $T \in \mathrm{M}_{2 n, k}$, we let

$$
r_{i j}^{2}(T)=\left(T_{i}, T_{j}\right)
$$

where $T_{i}$ and $T_{j}$ are the $i$ th and the $j$ th column of $T$, respectively. Let

$$
\mathcal{N}=\left\{T \in \mathrm{M}_{2 n, k}: r_{i j}^{2}(T)=0 \forall i, j\right\} .
$$

and $\mathcal{R}(\mathcal{N})$ be the algebra of regular functions on $\mathcal{N}$. Let $\mathrm{O}_{2 n} \times \mathrm{GL}_{k}$ act on $\mathcal{R}(\mathcal{N})$ by

$$
[(g, h) f](T)=f\left(g^{\mathrm{t}} T h\right), \quad g \in \mathrm{O}_{2 n}, h \in \mathrm{GL}_{k}, f \in \mathcal{R}(\mathcal{N}), T \in \mathcal{N} .
$$

Then as an $\mathrm{O}_{2 n} \times \mathrm{GL}_{k}$ module [How95],

$$
\mathcal{R}(\mathcal{N}) \cong \sum_{r(H) \leqslant k} \sigma_{2 n}^{H} \otimes \rho_{k}^{H} .
$$

Taking $U_{\mathrm{GL}_{k}}$ invariants gives

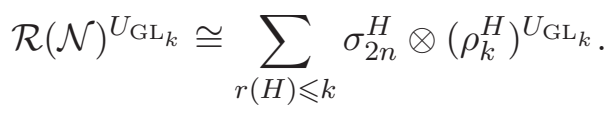

So this algebra contains one copy of each irreducible representation $\sigma_{2 n}^{H}$ of $\mathrm{O}_{2 n}$ with $r(H) \leqslant k$. We consider its subalgebra of $U_{\mathrm{GL}_{n}}$ invariants

$$
\mathcal{R}(\mathcal{N})^{U_{\mathrm{GL}_{n}} \times U_{\mathrm{GL}}} \cong \sum_{r(H) \leqslant k}\left(\sigma_{2 n}^{H}\right)^{U_{\mathrm{GL}}} \otimes\left(\rho_{k}^{H}\right)^{U_{\mathrm{GL}_{k}}}
$$

This algebra encodes information on the branching rule from $\mathrm{O}_{2 n}$ to $\mathrm{GL}_{n}$ for all representations $\sigma_{2 n}^{H}$ with $r(H) \leqslant k$. In view of this property, we call $\mathcal{R}(\mathcal{N})^{U_{\mathrm{GL}_{n}} \times U_{\mathrm{GL}_{k}}}$ a stable branching algebra for $\left(\mathrm{O}_{2 n}, \mathrm{GL}_{n}\right)$ (see [HTW04]).

\subsection{An index set for the basis}

The algebra $\mathcal{R}(\mathcal{N})^{U_{\mathrm{GL}_{n}} \times U_{\mathrm{GL}_{k}}}$ is an $A_{\mathrm{GL}_{n}} \times A_{\mathrm{GL}_{k}}$ module, and

$$
\mathcal{R}(\mathcal{N})^{U_{\mathrm{GL}_{n}} \times U_{\mathrm{GL}_{k}}=} \bigoplus_{r(D), r(E), r(H) \leqslant k} W_{(D, E), H} .
$$

Here for Young diagrams $D, E$ and $H$, all with at most $k$ rows, $W_{(D, E), H}$ is the $\psi_{n}^{D, E} \times \psi_{k}^{H}$-eigenspace of $A_{\mathrm{GL}_{n}} \times A_{\mathrm{GL}_{k}}$. Its dimension is equal to the multiplicity of the representation $\rho_{n}^{D, E}$ of $\mathrm{GL}_{n}$ in the representation $\sigma_{2 n}^{H}$ of $\mathrm{O}_{2 n}$, which is given by [HTW05a]

$$
\sum_{F \in \mathcal{E}_{k}, r(G) \leqslant k} c_{F, D}^{G} c_{G, E}^{H} .
$$

Let $\Omega(D, E, H)$ be the set defined in (5.2). Since the number of elements in $\Omega(D, E, H)$ coincides with $\operatorname{dim} W_{(D, E), H}$, we use it to index a basis for $W_{(D, E), H}$.

\subsection{The highest weight vector $\eta_{\left(F, G, T_{1}, T_{2}\right)}$}

In this subsection, we fix $\left(F, G, T_{1}, T_{2}\right) \in \Omega(D, E, H)$ and construct a $\mathrm{GL}_{n} \times \mathrm{GL}_{k}$ highest weight vector $\eta_{\left(F, G, T_{1}, T_{2}\right)}$ in the polynomial algebra $\mathcal{P}\left(\mathrm{M}_{2 n, k}\right)$. The restrictions of all such highest weight vectors to $\mathcal{N}$ form a basis for $W_{(D, E), H}$. 


\section{R. Howe And S. T. LeE}

Let the standard coordinates on $\mathrm{M}_{2 n, k}$ be given by

$$
\left(\begin{array}{cccc}
z_{11} & z_{12} & \cdots & z_{1 k} \\
\vdots & \vdots & & \vdots \\
z_{n 1} & z_{n 2} & \cdots & z_{n k} \\
w_{11} & w_{12} & \cdots & w_{1 k} \\
\vdots & \vdots & & \vdots \\
w_{n 1} & w_{n 2} & \cdots & w_{n k}
\end{array}\right)
$$

For $1 \leqslant a, b \leqslant k$, we also let

$$
\mu_{a b}=\sum_{i=1}^{n}\left(z_{i a} w_{n-i+1, b}-z_{i b} w_{n-i+1, a}\right) .
$$

We shall also use the notation related to $D, E, F, G, H, T_{1}$ and $T_{2}$ defined in $\S 5.5$. Let $\boldsymbol{\sigma}=\left(\sigma_{1}, \ldots\right.$, $\left.\sigma_{r}\right) \in S_{d_{1}} \times \cdots \times S_{d_{r}}$ and $\tau=\left(\tau_{1}, \ldots, \tau_{s}\right) \in S_{e_{1}} \times \cdots \times S_{e_{s}}$. For each $1 \leqslant i \leqslant u$, let

$$
p_{i}=g_{i}-f_{i},
$$

and $Z_{i}(\boldsymbol{\sigma})$ be the $p_{i} \times h_{i}$ matrix

$$
Z_{i}(\boldsymbol{\sigma})=\left(\begin{array}{c}
Z_{i 1}\left(\sigma_{1}\right) \\
Z_{i 2}\left(\sigma_{2}\right) \\
\vdots \\
Z_{i r}\left(\sigma_{r}\right)
\end{array}\right)
$$

where, for each $1 \leqslant j \leqslant r$,

$$
Z_{i j}\left(\sigma_{j}\right)=\left(\begin{array}{cccc}
z_{\sigma_{j}\left(a_{i+1, j}^{(1)}+1\right), 1} & z_{\sigma_{j}\left(a_{i+1, j}^{(1)}+1\right), 2} & \cdots & z_{\sigma_{j}\left(a_{i+1, j}^{(1)}+1\right), h_{i}} \\
z_{\sigma_{j}\left(a_{i+1, j}^{(1)}+2\right), 1} & z_{\sigma_{j}\left(a_{i+1, j}^{(1)}+2\right), 2} & \cdots & z_{\sigma_{j}\left(a_{i+1, j}^{(1)}+2\right), h_{i}} \\
\vdots & \vdots & & \vdots \\
z_{\sigma_{j}\left(a_{i, j}^{(1)}\right), 1} & z_{\sigma_{j}\left(a_{i, j}^{(1)}\right), 2} & \cdots & z_{\sigma_{j}\left(a_{i, j}^{(1)}\right), h_{i}}
\end{array}\right),
$$

and, for $1 \leqslant i \leqslant v$, let

$$
q_{i}=h_{i}-g_{i}
$$

and let $W_{i}(\boldsymbol{\tau})$ be the $q_{i} \times h_{i}$ matrix

$$
W_{i}(\boldsymbol{\tau})=\left(\begin{array}{c}
W_{i 1}\left(\tau_{1}\right) \\
W_{i 2}\left(\tau_{2}\right) \\
\vdots \\
W_{i s}\left(\tau_{s}\right)
\end{array}\right)
$$

where, for each $1 \leqslant l \leqslant s, W_{i l}\left(\tau_{l}\right)$ is the $m_{i l}^{(2)} \times h_{i}$ matrix

$$
W_{i l}\left(\tau_{l}\right)=\left(\begin{array}{cccc}
w_{\tau_{l}\left(a_{i+1, l}^{(2)}+1\right), 1} & w_{\tau_{l}\left(a_{i+1, l}^{(2)}+1\right), 2} & \cdots & w_{\tau_{l}\left(a_{i+1, l}^{(2)}+1\right), h_{i}} \\
w_{\tau_{l}\left(a_{i+1, l}^{(2)}+2\right), 1} & w_{\tau_{l}\left(a_{i+1, l}^{(2)}+2\right), 2} & \cdots & w_{\tau_{l}\left(a_{i+1, l}^{(2)}+2\right), h_{i}} \\
\vdots & \vdots & & \vdots \\
w_{\tau_{l}\left(a_{i, l}^{(2)}\right), 1} & w_{\tau_{l}\left(a_{i, l}^{(2)}\right), 2} & \cdots & w_{\tau_{l}\left(a_{i, l}^{(2)}\right), h_{i}}
\end{array}\right) .
$$


We also let, for $1 \leqslant m \leqslant k$,

$$
[\mu]_{m}=\left(\begin{array}{cccc}
\mu_{11} & \mu_{12} & \cdots & \mu_{1 m} \\
\mu_{21} & \mu_{22} & \cdots & \mu_{2 m} \\
\vdots & \vdots & & \vdots \\
\mu_{m 1} & \mu_{m 2} & \cdots & \mu_{m m}
\end{array}\right)
$$

Finally, we let

$$
\begin{aligned}
\eta_{\left(F, G, T_{1}, T_{2}\right)}= & \sum_{\substack{\boldsymbol{\sigma} \in S_{d_{1}} \times \cdots \times S_{d_{r}} \\
\boldsymbol{\tau} \in S_{e_{1}} \times \cdots \times S_{e_{s}}}}\left(\prod_{i=1}^{r} \operatorname{sgn}\left(\sigma_{i}\right)\right)\left(\prod_{j=1}^{s} \operatorname{sgn}\left(\tau_{j}\right)\right) \\
& \times\left[\prod_{i=1}^{v} \operatorname{Pfaff}\left(\begin{array}{c|c|c}
{[\mu]_{h_{i}}} & {\left[Z_{i}(\boldsymbol{\sigma})\right]^{\mathrm{t}}} & {\left[W_{i}(\boldsymbol{\tau})\right]^{\mathrm{t}}} \\
\hline-Z_{i}(\boldsymbol{\sigma}) & 0 & 0 \\
\hline-W_{i}(\boldsymbol{\sigma}) & 0 & 0
\end{array}\right)\right] .
\end{aligned}
$$

This is a $\mathrm{GL}_{n} \times \mathrm{GL}_{k}$ highest weight vector of weight $\psi_{n}^{D, E} \times \psi_{k}^{H}$.

\subsection{The leading monomial of $\eta_{\left(F, G, T_{1}, T_{2}\right)}$}

Let

$$
S=\left\{z_{i j}, w_{i j}: 1 \leqslant i, j \leqslant k\right\} \cup\left\{\mu_{i j}: 1 \leqslant i<j \leqslant k\right\}
$$

and $V=\operatorname{Span}(S)$. Then $S$ is an algebraically independent subset of $\mathcal{P}\left(\mathrm{M}_{2 n, k}\right)$, so the subalgebra of $\mathcal{P}\left(\mathrm{M}_{2 n, k}\right)$ generated by $S$ is a polynomial algebra. We denote this subalgebra by $\mathcal{P}(V)$. We observe that the highest weight vector $\eta_{\left(F, G, T_{1}, T_{2}\right)}$ belongs to this subalgebra $\mathcal{P}(V)$. In this subsection, we introduce a monomial ordering $\tau_{3}$ in $\mathcal{P}(V)$ and compute the leading monomial of the highest weight vector $\eta_{\left(F, G, T_{1}, T_{2}\right)}$ with respect to this ordering.

The monomial ordering $\tau_{3}$ is defined to be the graded lexicographic order [CLO97] such that

$$
\begin{aligned}
\mu_{12} & >\mu_{13}>\cdots>\mu_{1 k}>\mu_{23}>\mu_{24}>\cdots>\mu_{k-1, k} \\
& >z_{11}>z_{12}>\cdots>z_{1 k}>z_{21}>\cdots>z_{k k} \\
& >w_{11}>w_{12}>\cdots>w_{1 k}>w_{21}>\cdots>w_{k k} .
\end{aligned}
$$

Lemma 6.5.1. The leading monomial of $\eta_{\left(F, G, T_{1}, T_{2}\right)}$ with respect to $\tau_{3}$ is given by

$$
\left(\prod_{\mathbf{b}_{1} \in T_{1}} z_{c_{1}\left(\mathbf{b}_{1}\right) a_{1}\left(\mathbf{b}_{1}\right)}\right)\left(\prod_{\mathbf{b}_{2} \in T_{2}} w_{c_{2}\left(\mathbf{b}_{2}\right) a_{2}\left(\mathbf{b}_{2}\right)}\right)\left(\prod_{j=1}^{t} \prod_{i=1}^{f_{j} / 2} \mu_{2 i-1,2 i}\right)
$$

where:

(i) for each box $\mathbf{b}_{1}$ in $T_{1}, a_{1}\left(\mathbf{b}_{1}\right)$ is the row of $G$ in which the box $\mathbf{b}_{1}$ lies and $c_{1}\left(\mathbf{b}_{1}\right)$ is the entry in $\mathbf{b}_{1}$; and

(ii) for each box $\mathbf{b}_{2}$ in $T_{2}, a_{2}\left(\mathbf{b}_{2}\right)$ is the row of $H$ in which the box $\mathbf{b}_{2}$ lies and $c_{2}\left(\mathbf{b}_{2}\right)$ is the entry in $\mathbf{b}_{2}$.

The proof is similar to the proof of Lemma 9.4.1 in [HL06b]. We note that the leading monomials of the $\eta_{\left(F, G, T_{1}, T_{2}\right)}$ are all distinct, so they form a linearly independent set.

Theorem 6.5.2. Let $\tilde{\eta}_{\left(F, G, T_{1}, T_{2}\right)}$ denote the restriction of $\eta_{\left(F, G, T_{1}, T_{2}\right)}$ to $\mathcal{N}$. Then the set

$$
\bigcup_{r(D), r(E), r(H) \leqslant k}\left\{\tilde{\eta}_{\left(F, G, T_{1}, T_{2}\right)}:\left(F, G, T_{1}, T_{2}\right) \in \Omega(D, E, H)\right\}
$$

is a basis for $\mathcal{R}(\mathcal{N})^{U_{\mathrm{GL}_{n}} \times U_{\mathrm{GL}_{k}}}$. 


\section{BASES FOR SOME RECIPROCITY ALGEBRAS III}

\section{REFERENCES}

CLO97 D. Cox, J. Little and D. O'Shea, Ideals, varieties, and algorithms. An introduction to computational algebraic geometry and commutative algebra, second edition, Undergraduate Texts in Mathematics, vol. 13 (Springer, New York, 1997).

Fu97 W. Fulton, Young tableaux, London Mathematical Society Student Texts, vol. 35 (Cambridge University Press, Cambridge, 1997).

GW98 R. Goodman and N. R. Wallach, Representations and invariants of the classical groups, Encyclopedia of Mathematics and its Applications, vol. 68 (Cambridge University Press, Cambridge, 1998).

How95 R. Howe, Perspectives on invariant theory, in The Schur Lectures, eds I. Piatetski-Shapiro and S. Gelbart, Israel Mathematical Conference Proceedings, vol. 8 (Bar-Ilan University, Ramat Gan, 1995), 1-182.

HJLTW06 R. Howe, S. Jackson, S. Lee, E.-C. Tan and J. Willenbring, Toric degeneration of branching algebras, Preprint (2006).

HL06a R. Howe and S. Lee, Bases for some reciprocity algebras I, Trans. Amer. Math. Soc., to appear.

HL06b R. Howe and S. Lee, Bases for some reciprocity algebras II, Adv. Math. 206 (2006), 145-210.

HTW04 R. Howe, E.-C. Tan and J. Willenbring, Reciprocity algebras and branching for classical symmetric pairs, Preprint (2004), math.RT/0407467.

HTW05a R. Howe, E.-C. Tan and J. Willenbring, Stable branching rules for classical symmetric pairs, Trans. Amer. Math. Soc. 357 (2005), 1601-1626.

HTW05b R. Howe, E.-C. Tan and J. Willenbring, A basis for the $\mathrm{GL}_{n}$ tensor product algebra, Adv. Math. 196 (2005), 531-564.

RS90 L. Rubbiano and M. Sweedler, Subalgebra bases, in Commutative algebra, Salvador, 1988, Lecture Notes in Mathematics, vol. 1430 (Springer, Berlin, 1990), 61-87.

Stu96 B. Sturmfels, Gröbner bases and convex polytopes, University Lecture Series, vol. 8 (American Mathematical Society, Providence, RI, 1996).

Roger Howe howe@math.yale.edu

Department of Mathematics, Yale University, New Haven, CT 06520-8283, USA

Soo Teck Lee matleest@nus.edu.sg

Department of Mathematics, National University of Singapore, 2 Science Drive 2, Singapore 117543, Singapore 